\title{
Proprioceptive Accuracy in Immersive Virtual Reality: A Developmental Perspective
}

Irene Valori, Phoebe E. McKenna-Plumley, Rena Bayramova, Claudio Zandonella Callegher, Gianmarco Altoè \& Teresa Farroni

\section{Supplemental Materials}

\author{
Edited by \\ Claudio Zandonella Callegher* \& Gianmarco Altoè \\ *e-mail address \\ claudiozandonella@gmail.com
}

January 15,2020 


\section{Contents}

\begin{tabular}{lll}
\hline & Introduction & 1
\end{tabular}

1.1 Statistical Approach . . . . . . . . . . . . . . . . . . . . . . . . 1

1.2 Statistical Analysis . . . . . . . . . . . . . . . . . . . . . . . . 2

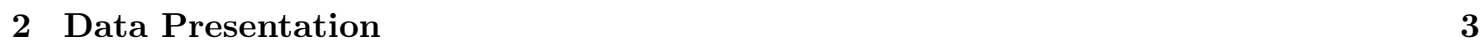

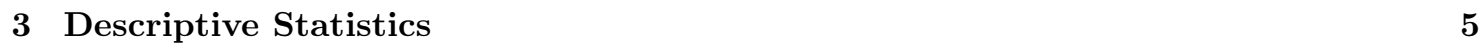

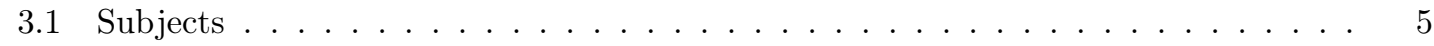

3.2 Number of Observations . . . . . . . . . . . . . . . . . . . . . . . . 5

3.3 Amplitude ............................. 6

3.3.1 Variability of Amplitude across conditions and Age groups. . . . . . . . . . 7

3.4 Self-turn Error . . . . . . . . . . . . . . . . . . . 8

\begin{tabular}{|l|l|}
\hline Model Comparison & 11
\end{tabular}

4.1 Models Specification . . . . . . . . . . . . . . . . . . . . . . . . . 11

4.1 .1 Generalized Linear Model . . . . . . . . . . . . . . . . . . . . . . . . . . . . 12

4.2 Models Priors . . . . . . . . . . . . . . . . . . . . . . . . . . . . . . . . . . . . . . 12

4.3 Models Estimation . . . . . . . . . . . . . . . . . . . . . . . . . . . . 13

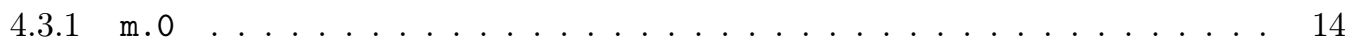

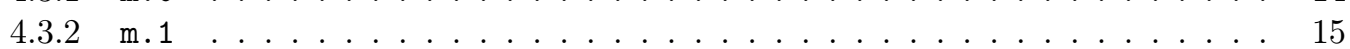

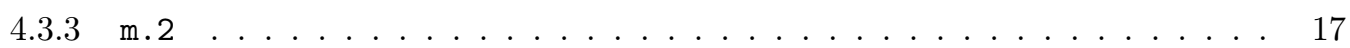

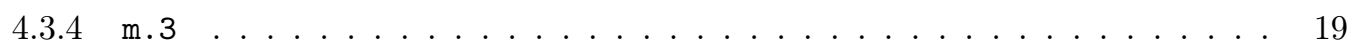

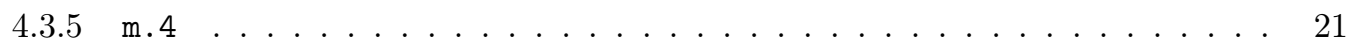

$4.3 .6 \mathrm{~m} .5$. . . . . . . . . . . . . . . . . . . . . . . 23

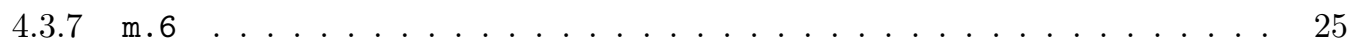

4.4 Model Comparison . . . . . . . . . . . . . . . . . . . . . . . . 27

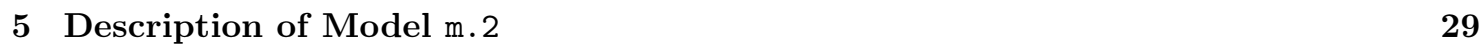

5.1 Model Parameters . . . . . . . . . . . . . . . . . . . . . . . . . 29

5.1 .1 Amplitude . . . . . . . . . . . . . . . . . . . . . . . 30

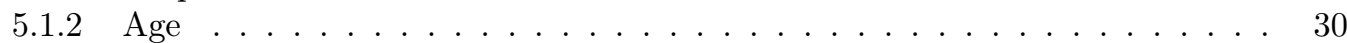

$5.1 .3 \quad$ Interaction between Perception and Environment . . . . . . . . . . . . . . . 32

5.2 Effect Sizes . . . . . . . . . . . . . . . . . . . . . . 33

5.3 Model Fit . . . . . . . . . . . . . . . . . . . . . . . . . . . . . . . . . 34

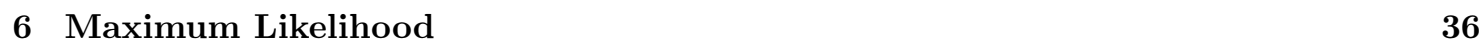

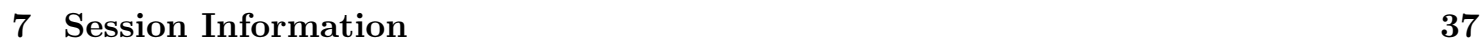

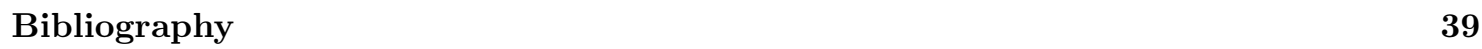




\section{List of Figures}

$1 \quad$ Amplitude distribution $\ldots \ldots \ldots \ldots \ldots \ldots \ldots$

2 Variability of Amplitude according to conditions and Age groups . . . . . . . . . . 7

$3 \quad$ Frequency of Self-turn errors $\ldots \ldots \ldots \ldots \ldots$

$4 \quad$ Distributions of Self-turn errors . . . . . . . . . . . . . . . . . . . . . . . . 10

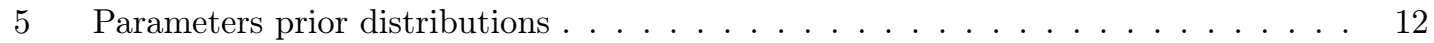

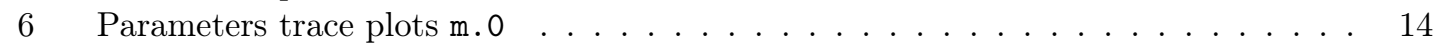

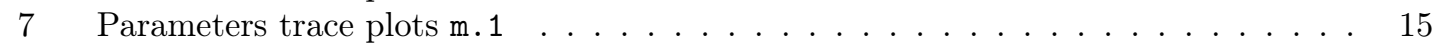

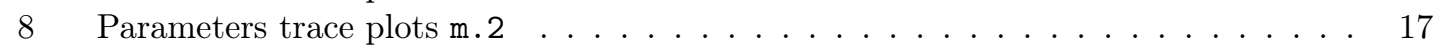

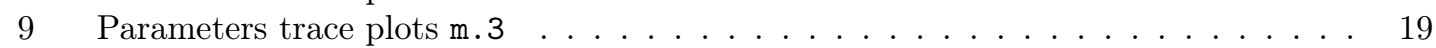

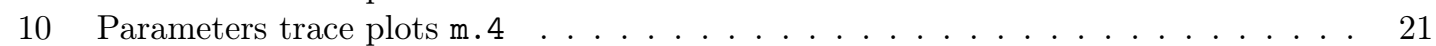

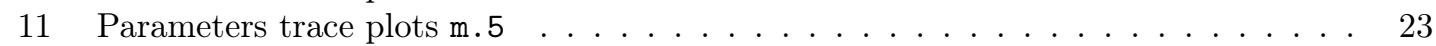

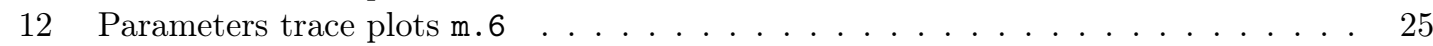

13 Predicted mean of Self-turn error according to Amplitude.st . . . . . . . . . . . . . 30

14 Distributions of the predicted means of Self-turn error according to Age . . . . . . 31

15 Distributions of the predicted means of Self-turn error according to the experimental

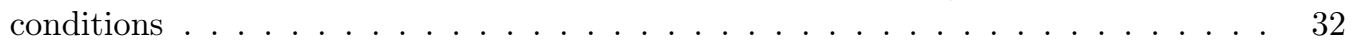

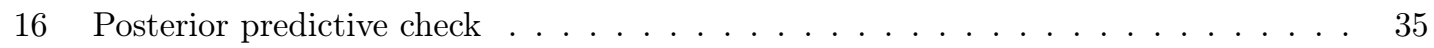

\section{List of Tables}

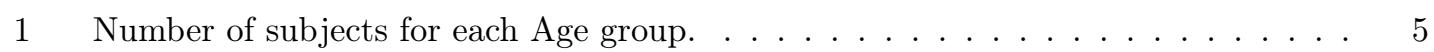

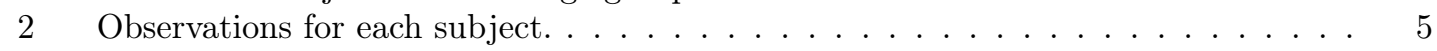

$3 \quad$ Number of observations according to Age groups and conditions. . . . . . . . . . 6

$4 \quad$ Descriptive statistics $\ldots \ldots \ldots \ldots \ldots \ldots$

5 Model formulas . . . . . . . . . . . . . . . . . . . . . . . . . . . . . . 11

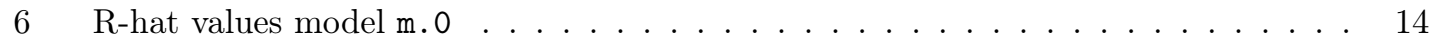

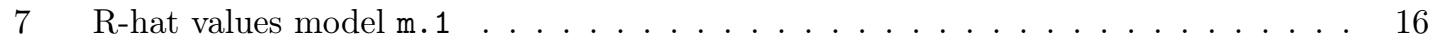

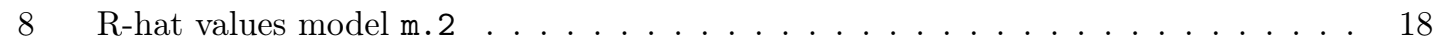

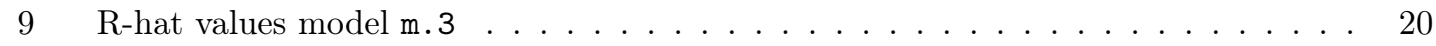

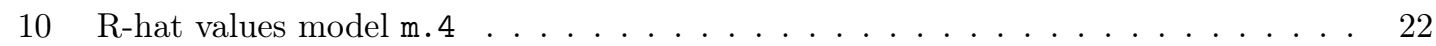

11 R-hat values model m.5 . . . . . . . . . . . . . . . . . . . . . 24

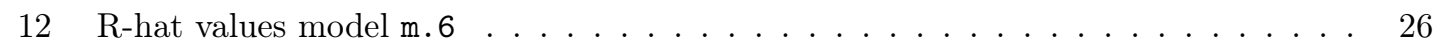

13 WAIC model comparison . . . . . . . . . . . . . . . . . . . . 27

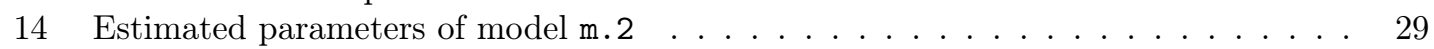

$15 \quad$ Predicted means and differences of Self-turn error according to age. . . . . . . . . . 31

16 Predicted means and differences of Self-turn error according experimental conditions. 33

17 Effect size as the ratio of the scores $\ldots \ldots \ldots \ldots \ldots \ldots \ldots$

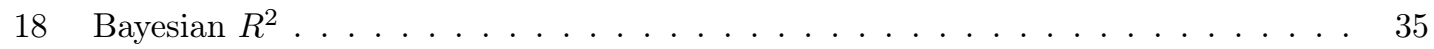

19 Comparison between Bayesian m.2 and Maximum-Likelihood glm.2 models . . . 36 


\section{Introduction}

In this report the statistical analyses of the article "Proprioceptive Accuracy in Immersive Virtual Reality: A Developmental Perspective" are presented. The aim of the study is to investigate the extent to which the reliability of visual information aids proprioceptive-based self-motion accuracy across the developmental lifespan. Moreover, it explores whether Immersive Virtual Reality (IVR) environments, compared to equivalent real environments, affect proprioceptive accuracy.

Here, we will focus only on the statistical analyisis. For theoretical aspects, study aims, experiment design, and results interpretation the reader can refer directly to the article.

\subsection{Statistical Approach}

In order to evaluate the research hypotheses about how Age, Perception, and Environment interact to affect self-motion accuracy, a model comparison approach was used.

Model comparison allows for the selection of the most plausible models given the data and a set of candidate models (McElreath, 2016). Models are compared in terms of statistical evidence (i.e. support by the data) using information criteria (Wagenmakers \& Farrell, 2004), which enables the evaluation of models considering the trade-off between parsimony and goodness-of-fit (Vandekerckhove, Matzke, \& Wagenmakers, 2015): as the complexity of the model increases (i.e. more parameters) the fit to the data increases as well but generalizability (i.e. ability to predict new data) decreases. The researchers' aim is to find the right balance between fit and generalizability in order to describe, with a statistical model, the important feature of the studied phenomenon, but not the random noise of the observed data. Information criteria provide an estimate of the average deviance (i.e. error) of the model's ability to predict new data, thus lower values are interpreted as indications of a better model (McElreath, 2016).

Given the complex structure of the data, Bayesian Generalized Mixed-Effects Models are used. Specifically, data are characterized by: (1) a continuous non-normally distributed dependent variable (i.e. rotation error); (2) a between-subject factor (i.e. age); (3) within-subject factors (i.e. Perception and Environment); (4) a quantitative independent variable (i.e. rotation Amplitude).

- Mixed-Effects Models. Mixed-Effects Models allow us to take into account the repeated measures design of the experiment (i.e. observations nested within subjects). Thus, participants are treated as random effects, with random intercepts that account for the interpersonal variability, whereas the other variables are considered as fixed effects.

- Generalized Linear Models. Generalized linear models were used considering the Gamma distribution, with logarithmic link function, as the probability distribution of the dependent variable. Generalized linear models allow to model non-normally distributed data using appropriate probability distributions that reflect the characteristics of the data (Fox, 2016). Selecting an appropriate probability distribution provides better fit to the data and more reliable results (Lo \& Andrews, 2015). Gamma distribution is advised in the case of positively skewed, non-negative data, when the variances are expected to be proportional to the square of the means (Ng \& Cribbie, 2017). These conditions are respected by our dependent variable: we only have positive values, with a positive skewed distribution, and we expect a greater variability of the possible results as the model predicted mean increases (i.e. a greater dispersion of subjects' scores when greater mean values are predicted by the model).

- Bayesian approach. The Bayesian approach is a valid alternative to the traditional frequentist approach (Gelman et al., 2013, Kruschke \& Liddell, 2018) and in the case of mixedeffects models it allows for the accurate estimation of complex models that otherwise would fail to converge (i.e. unreliable results) in a traditional frequentist approach (Bolker et al., 2009 Fong, Rue, \& Wakefield, 2010). Without going into philosophical reasons, which are beyond the scope of the present paper (if interested consider Romeijn \& van de Schoot, 2008), Bayesian inference has some unique elements that make the meaning and interpretation of the results different from the classical frequentist approach (Etz \& Vandekerckhove, 
2018). In particular, in the Bayesian approach parameters are estimated using probability distributions (i.e. a range of possible values) and not a single point estimate (i.e. a single value). Bayesian inference has three main ingredients (van de Schoot et al., 2014): (1) Priors, the probability distributions of possible parameter values considering the information available before conducting the experiment; (2) Likelihood, the information given by the observed data about the probability distributions of possible parameter values; (3) Posteriors, the resulting probability distributions of possible parameter values, obtained by combining Priors and Likelihood through Bayes' Theorem. As a result, the Bayesian approach assesses the variability (i.e. uncertainty) of parameter estimates and provides associated inferences via 95\% Bayesian Credible Intervals (BCIs): the range of most credible parameter values given the prior distribution and the observed data. Thus, the Bayesian approach allows for the description of the phenomenon of interest through probabilistic statements, rather than a series of simplified reject/not-reject dichotomous decisions typically used in the null hypothesis significance testing approach (McElreath, 2016).

Analysis were conducted with the $\mathrm{R}$ software version 3.6.1 ( $\mathrm{R}$ Core Team, 2018). Models were estimated using the R package brms (Bürkner, 2017) which is based on STAN programming language (Stan Development Team, 2017a, 2017b) and employs the No-U-Turn Sampler (NUTS; Hoffman \& Gelman, 2014), an extension of Hamiltonian Monte Carlo (Neal, 2012). The basic idea of estimating parameters through a sampling procedure is to iteratively poll possible parameter values from pre-specified prior distributions. Parameter values that support the data are rewarded, whereas those values that do not support the data are penalized. After a large number of iterations convergence upon those model parameters that optimally represent the data is obtained. Iterations of the estimation procedure are split among independent chains (i.e. independent sampling processes) to ensure that the model reliably converges on the same parameter values. Since each chain has a random starting point, it requires a certain number of iterations before reaching an optimal convergence. Thus, for each chain the initial samples, known as "warm-up", are discarded and only the subsequent sampling part is used for inference purposes.

\subsection{Statistical Analysis}

The analysis is divided into different sections:

- Section 2, We introduce the dataset with a brief description of each variable.

- Section 3. We present the descriptive statistics of our dataset.

- Section 4. We formalize the models and present the prior and the diagnostic criteria for convergence of parameters estimation. Finally, we give the results of the model comparison.

- Section 5. The most plausible model is interpreted using the estimated posterior parameter distributions. Main effects and interaction effects are considered using planned comparison and graphical representations of the predicted values by model. Finally, we evaluate the model fit considering $R^{2}$ value and model Posterior Predictive check.

- Section 6. We present a separate set of analyses where all models are estimated with the traditional maximum likelihood approach using the R package lme4 (Bates, Mächler, Bolker, \& Walker, 2014). In several cases, convergence is not reached (i.e. unreliable results). Overall, estimated model parameters are very similar to those produced by the Bayesian approach. 


\section{Data Presentation}

In the present dataset we have 13 variables:

1. Id. Each subject has an unique Id ranging from 1 to 49 .

2. Years. The age in years of each subject.

3. Age. We have three different age groups: Adults 18-45 years; Older Children subjects between 9 and 15 years old; Young Children subjects 4 and 8 years old and younger.

4. Environment. The two environmental conditions Reality $(R)$ and Immersive Virtual Reality $(I V R)$.

5. Perception. The three sensory conditions Proprioception $(P)$,Vision $(V)$, and Vision + Proprioception (VP).

6. Condition. A summary index between 1 and 6 to specify the experimental condition.

- 1) Reality and Proprioception

- 2) Reality and Vision

- 3) Reality and Vision + Proprioception

- 4) IVR and Proprioception

- 5) IVR and Vision

- 6) IVR and Vision + Proprioception

7. Direction. The direction of the passive rotation imposed by the experimenter on the subject, Right $(R)$ or Left $(L)$.

8. Amplitude. The amplitude of the passive rotation imposed by the experimenter on the subject, Big approximately 180 degrees or Small approximately 90 degrees.

9. Start.Position. Position from which the experimenter started the passive rotation.

10. End.Position. Position where the experimenter stopped the passive rotation.

11. Return.Position. Position in which the subject stopped the active self-turn.

12. Angle. The actual angle of the passive rotation imposed by the experimenter on the subject.

13. Error. The absolute error committed by the subject in the Self-turn task, computed as the absolute rotation difference between Start.Position and Return.Position.

Below we report the structure of the dataset.

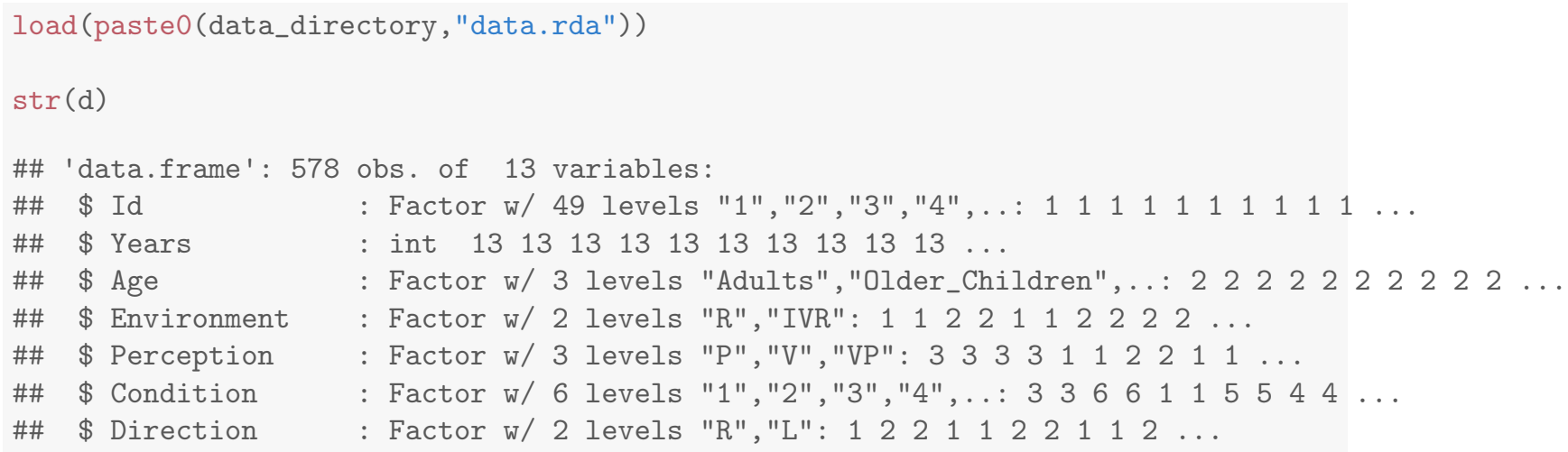




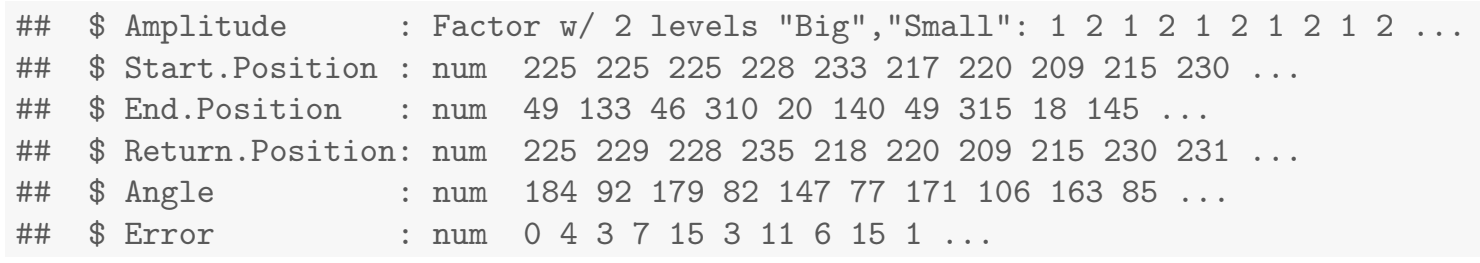




\section{Descriptive Statistics}

\subsection{Subjects}

Considering the Age of the subjects we have 23 Adults subjects, 13 Older Children subjects, and 13 Young Children subjects (11).

Table 1: Number of subjects for each Age group.

\begin{tabular}{ccc}
\hline age & n.subjects & percentages \\
\hline Adults & 23 & 0.47 \\
Older Children & 13 & 0.27 \\
Young Children & 13 & 0.27 \\
Total & 49 & 1.00 \\
\hline
\end{tabular}

\subsection{Number of Observations}

Out of the 49 participants, 43 subjects completed the task in all 12 conditions, 4 subjects completed 11 conditions, 1 subject completed 10 conditions, and 1 subject completed 8 conditions (2). Thus, the final data consist of 578 observations nested in 49 subjects.

Table 2: Observations for each subject.

\begin{tabular}{cc}
\hline n.subjects & n.observations \\
\hline 1 & 8 \\
1 & 10 \\
4 & 11 \\
43 & 12 \\
\hline
\end{tabular}

The number of observations according to age groups and experimantal conditions is reported in 3 . 
Table 3: Number of observations according to Age groups and conditions.

\begin{tabular}{|c|c|c|c|c|}
\hline & Proprioception & Vision & Vision + Proprioception & Total \\
\hline \multicolumn{5}{|l|}{ Reality } \\
\hline Adults & 46 & 45 & 46 & 137 \\
\hline Older Children & 26 & 26 & 25 & 77 \\
\hline Young Children & 25 & 25 & 25 & 75 \\
\hline Total & 97 & 96 & 96 & 289 \\
\hline \multicolumn{5}{|l|}{ IVR } \\
\hline Adults & 44 & 48 & 46 & 138 \\
\hline Older Children & 26 & 26 & 26 & 78 \\
\hline Young Children & 24 & 25 & 24 & 73 \\
\hline Total & 94 & 99 & 96 & 289 \\
\hline \multicolumn{5}{|l|}{ Total } \\
\hline Adults & 90 & 93 & 92 & 275 \\
\hline Older Children & 52 & 52 & 51 & 155 \\
\hline Young Children & 49 & 50 & 49 & 148 \\
\hline Total & 191 & 195 & 192 & 578 \\
\hline
\end{tabular}

Note: $\quad$ IVR $=$ Immersive Virtual Reality. $n_{\text {subjects }}=49 ; n_{\text {observations }}=578$

\subsection{Amplitude}

For each condition, the task aimed to include two rotations of different Amplitude, one 180 degree (Big) rotation and one 90 degree $($ Small) rotation. However, as we can see in 1, there is a great variability in the actual rotation (reported in the variable Angle). Thus, we considered Amplitude as a continuous variable and not as a dichotomous categorical variable.

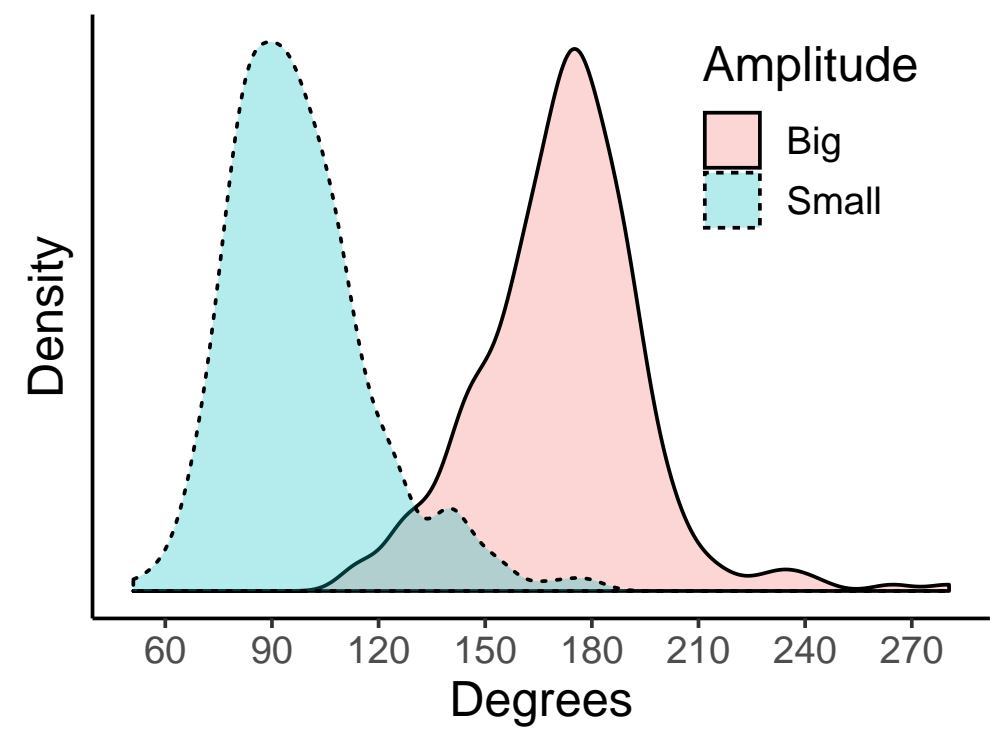

Figure 1: Amplitude distribution of the actual Self-turn rotation task (i.e. task difficulty; $\left.n_{\text {subjects }}=49 ; n_{\text {observations }}=578\right)$. 
Moreover, to obtain interpretable results in the analyses, the Amplitude variable was standardized (i.e., Z-scores were obtained).

d\$Amplitude. st<- (d\$Angle-mean (d\$Angle))/sd (d\$Angle)

\subsubsection{Variability of Amplitude across conditions and Age groups}

In 2 the distribution of the actual Self-turn rotation task is presented in the different experimental conditions according to Age groups. It is possible to observe that distributions are slightly different, but all of them cover appropriately the same range of values. Considering the Amplitude as a continuous variable and not as a dichotomous categorical variable guarantees that results are not influenced by the variability in the actual rotation between experimental conditions and Age groups.

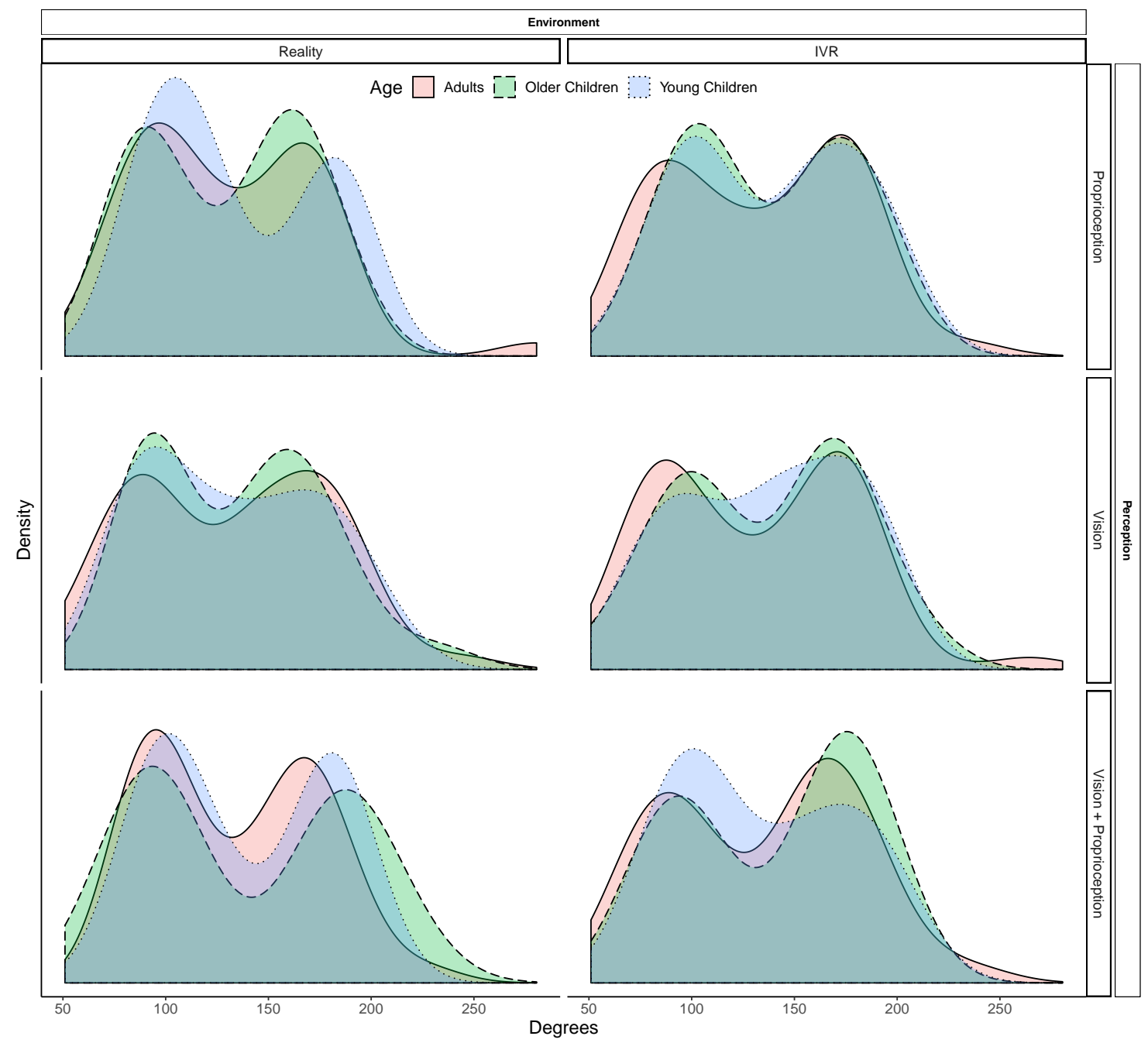

Figure 2: Amplitude distribution of the actual Self-turn rotation in the different experimental conditions according to Age group $\left(n_{\text {subjects }}=49 ; n_{\text {observations }}=578\right)$. 
Table 4: Descriptive statistics. Means and standard deviations of self-turn error according to Age and the experimental conditions

\begin{tabular}{|c|c|c|c|c|c|c|c|c|}
\hline & \multicolumn{2}{|c|}{ Proprioception } & \multicolumn{2}{|c|}{ Vision } & \multicolumn{2}{|c|}{ Vision + Proprioception } & \multicolumn{2}{|c|}{ Total } \\
\hline & Mean & SD & Mean & SD & Mean & SD & Mean & SD \\
\hline \multicolumn{9}{|l|}{ Reality } \\
\hline Adults & 16.2 & 8.6 & 9.8 & 12.6 & 6.1 & 4.1 & 10.7 & 6.0 \\
\hline Older Children & 19.6 & 10.5 & 14.0 & 18.2 & 6.7 & 3.6 & 13.5 & 7.3 \\
\hline Young Children & 30.6 & 22.4 & 8.2 & 5.2 & 20.7 & 20.5 & 19.8 & 9.0 \\
\hline Total & 20.9 & 15.0 & 10.5 & 12.9 & 10.1 & 12.5 & 13.9 & 8.0 \\
\hline \multicolumn{9}{|l|}{ IVR } \\
\hline Adults & 17.6 & 10.6 & 13.5 & 7.6 & 13.7 & 9.1 & 14.9 & 6.3 \\
\hline Older Children & 23.6 & 19.1 & 17.5 & 10.1 & 16.9 & 18.6 & 19.3 & 9.5 \\
\hline Young Children & 37.8 & 16.2 & 28.5 & 16.5 & 25.1 & 16.5 & 30.3 & 9.9 \\
\hline Total & 24.7 & 16.8 & 18.5 & 12.6 & 17.4 & 14.6 & 20.2 & 10.3 \\
\hline \multicolumn{9}{|l|}{ Total } \\
\hline Adults & 17.1 & 6.4 & 11.8 & 8.0 & 9.9 & 4.8 & 12.8 & 4.4 \\
\hline Older Children & 21.6 & 13.7 & 15.7 & 11.8 & 11.7 & 9.4 & 16.4 & 7.5 \\
\hline Young Children & 34.2 & 18.0 & 18.2 & 7.9 & 23.4 & 15.8 & 25.3 & 7.7 \\
\hline Total & 22.8 & 14.1 & 14.5 & 9.3 & 13.9 & 11.3 & 17.1 & 8.0 \\
\hline
\end{tabular}

Note: $\quad$ IVR $=$ Immersive Virtual Reality. $n_{\text {subjects }}=49 ; n_{\text {observations }}=578$

\subsection{Self-turn Error}

Given that observations are not independent but nested within subjects, to compute descriptive statistics in each condition first the mean Self-turn error was obtained for each subject and then mean and standard deviation were computed.

In the present sample, the mean Self-turn error was 17.07 degrees $(S D=8.05)$.

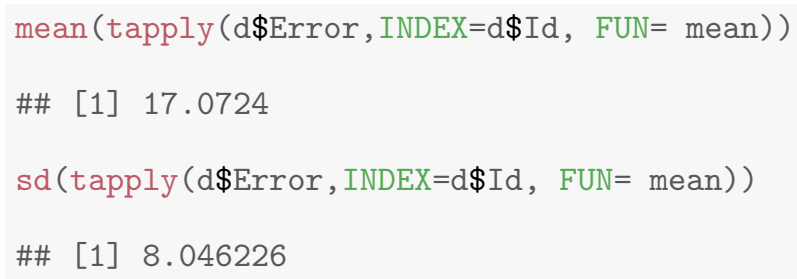

Mean and standard deviation according to Age groups and the different experimental conditions are reported in 4

The frequency of the observed values is reported in 3 Since Self-turn error is computed as the absolute difference between Start.Position and Return.Position, only positive values are possible and from visual inspection the dependent variable has an evident positive skewed distribution (skewness value $=2.49$ ). 


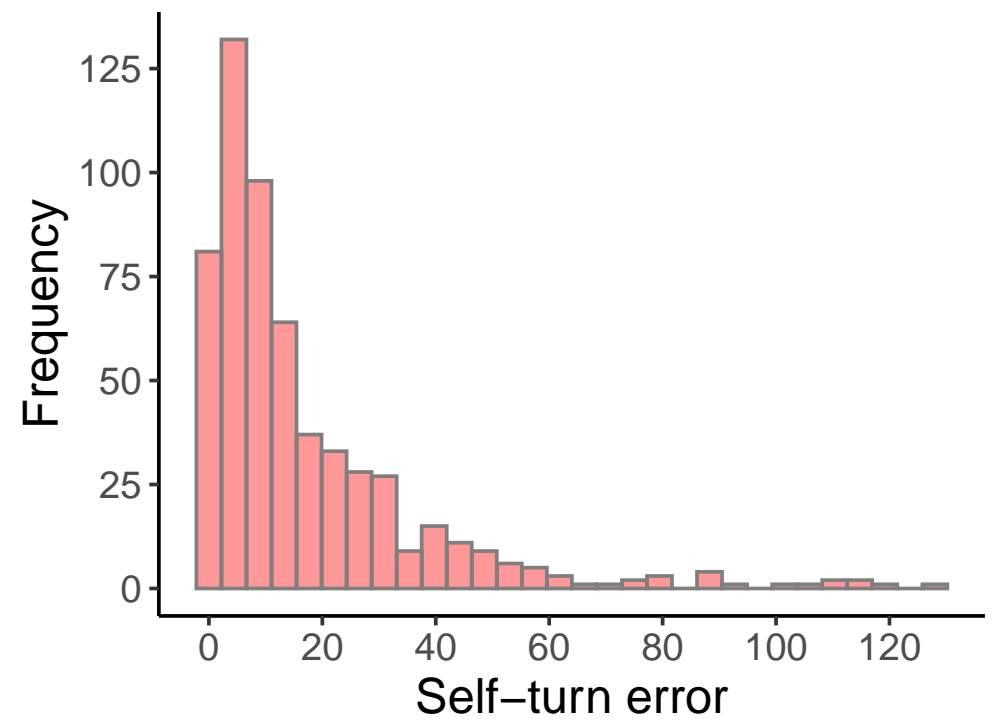

Figure 3: Frequency of the observed Self-turn errors $\left(n_{\text {subjects }}=49 ; n_{\text {observations }}=578\right)$.

Finally we represent in 4 the distribution of the observed Self-turn error according to age groups and the different experimental conditions. 


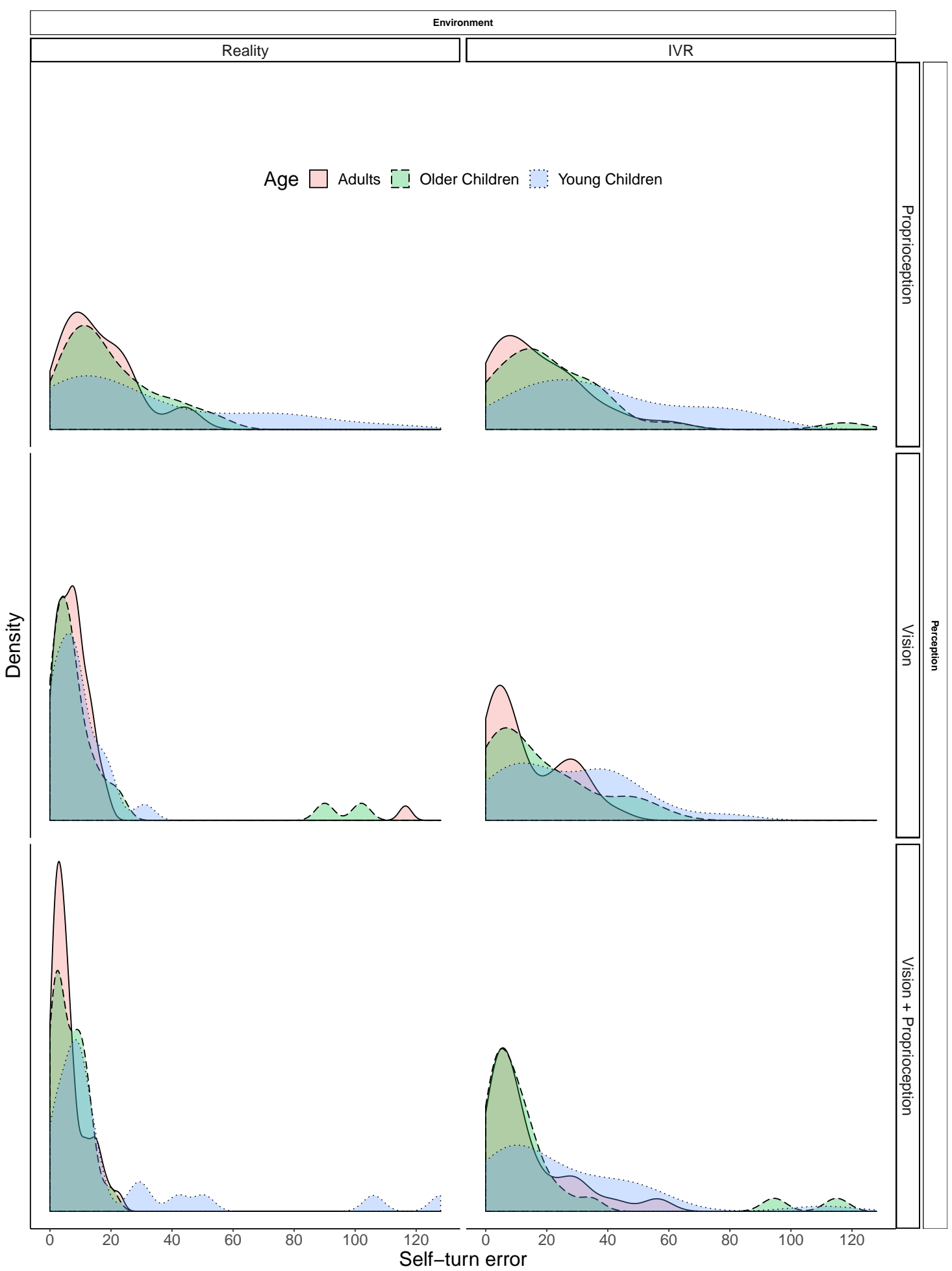

Figure 4: Distributions of the observed Self-turn errors in the different conditions according to Age $\left(n_{\text {subjects }}=49 ; n_{\text {observations }}=578\right)$. 


\section{Model Comparison}

In this section we first specify all the Bayesian Generalized Mixed-Effects Models used in the analysis. We formalize as statistical models the different research hypotheses, which include 2-way and 3-way interactions between independent variables. Subsequently we present the prior and the diagnostic criteria for convergence of parameters estimation. Finally, we give the results of the model comparison.

\subsection{Models Specification}

Seven different Bayesian Generalized Mixed-Effects Models were performed to analyse the data. In each model the dependent variable was the error in the self-turn task. The first model (m.0) was a baseline model considering the random effect of Id (i.e. interpersonal varibility) and the fixed effects of Direction (i.e. right or left rotation) and of Amplitude.st (i.e. amplitude of the rotation in degrees). This baseline model, that includes the effects of possible confounding variables, was used as a reference point to then evaluate the models that considered the effects of Age, Perception and Environment.

Below, the formula for each model is reported :

$$
\begin{aligned}
& m .0: \text { Error } \sim \text { Amplitude.st }+ \text { Direction }+(1 \mid I d) \\
& m .1: \text { Error } \sim \text { Age }+ \text { Perception }+ \text { Environment }+ \text { Amplitude.st }+ \text { Direction }+(1 \mid I d) \\
& m .2: \text { Error } \sim \text { Age }+ \text { Perception } * \text { Environment }+ \text { Amplitude.st }+ \text { Direction }+(1 \mid I d) \\
& m .3: \text { Error } \sim \text { Age } * \text { Environment }+ \text { perception }+ \text { Amplitude.st }+ \text { Direction }+(1 \mid I d) \\
& m .4: \text { Error } \sim \text { Age } * \text { Perception }+ \text { Environment }+ \text { Amplitude.st }+ \text { Direction }+(1 \mid I d) \\
& m .5: \text { Error } \sim(\text { Age }+ \text { Perception }+ \text { Environment })^{2}+\text { Amplitude.st }+ \text { Direction }+(1 \mid I d) \\
& m .6: \text { Error } \sim(\text { Age }+ \text { Perception }+ \text { Environment })^{3}+\text { Amplitude.st }+ \text { Direction }+(1 \mid I d)
\end{aligned}
$$

\begin{tabular}{|c|c|c|c|c|c|}
\hline & \multirow[b]{2}{*}{ Model } & \multirow[b]{2}{*}{$\begin{array}{l}\text { Dependent } \\
\text { Variable }\end{array}$} & & \multicolumn{2}{|l|}{ Predictors } \\
\hline & & & & Fixed Effects & Random Effect \\
\hline Baseline & $\mathrm{m} .0$ & error & $\leftarrow$ & Amplitude.st + Direction & Id \\
\hline Additive & $\mathrm{m} .1$ & error & $\leftarrow$ & $\mathrm{m} .0+$ Age + Perception + Environment & Id \\
\hline $\begin{array}{r}2 \text {-way } \\
\text { Interactions }\end{array}$ & $\begin{array}{l}\mathrm{m} \cdot 2 \\
\mathrm{~m} \cdot 3 \\
\mathrm{~m} \cdot 4\end{array}$ & $\begin{array}{l}\text { error } \\
\text { error } \\
\text { error }\end{array}$ & $\begin{array}{l}\leftarrow \\
\leftarrow \\
\leftarrow\end{array}$ & $\begin{array}{l}\mathrm{m} .1+\text { Perception } \times \text { Environment } \\
\mathrm{m} .1+\text { Age } \times \text { Environment } \\
\mathrm{m} .1+\text { Age } \times \text { Perception }\end{array}$ & $\begin{array}{l}\text { Id } \\
\text { Id } \\
\text { Id }\end{array}$ \\
\hline $\begin{array}{r}\text { All 2-way } \\
\text { Interactions }\end{array}$ & m. 5 & error & $\leftarrow$ & $\begin{array}{l}\text { m. } 1+\text { Perception } \times \text { Environment }+ \\
\text { Age } \times \text { Environment }+ \text { Age } \times \text { Perception }\end{array}$ & Id \\
\hline $\begin{array}{r}\text { 3-way } \\
\text { Interactions }\end{array}$ & $\mathrm{m} .6$ & error & $\leftarrow$ & m.5 + Age $\times$ Perception $\times$ Environment & Id \\
\hline
\end{tabular}

In m. 1 the additive effects of Age, Perception and Environment were added to the baseline model. Single 2-way interactions were evaluated in models m.2, m.3, and m.4. In the model m.5 all the possible 2-way interactions between Age, Perception and Environment were considered together. Finally, in m. 6 also the 3-way interaction was added. Models are summarized in 5 .

Table 5: Model formulas 


\subsubsection{Generalized Linear Model}

We specified the Gamma distribution with logarithmic link function as the family distribution for our Bayesian Generalized Mixed-Effects Models. Given that there are some 0 values in the Self-turn error, in order to use the logarithmic link function we need first to add 1 to all the errors. This is done to avoid indefinite values (i.e. $\log (0)=-\infty$ ).

d\$Error 1<-d\$Error+1

\subsection{Models Priors}

All our models used default prior specification of the R package brms (Bürkner, 2017) that are considered to be only very weakly informative in order to influence results as little as possible, while providing at least some regularization to considerably improve convergence and sampling efficiency. This allows the posterior distributions to be mostly influenced by the observed data rather than by prior information.

In particular, for the population-level effects (i.e. fixed effects) brms uses an improper flat prior over the reals. Group-level effects (i.e. random effects) are restricted to be non-negative and, by default, have a half student-t prior with 3 degrees of freedom and a scale parameter of 10 student_t $(3,0,10)$. Intercept prior is set to be a student-t with 3 degrees of freedom, location parameter of 2 and scale parameter of 10 student_t $(3,2,10)$. Finally the prior for the shape parameter for the gamma distribution is set to gamma $(0.01,0.01)$.

In 5 the different prior distributions are presented.

Fixed effect prior

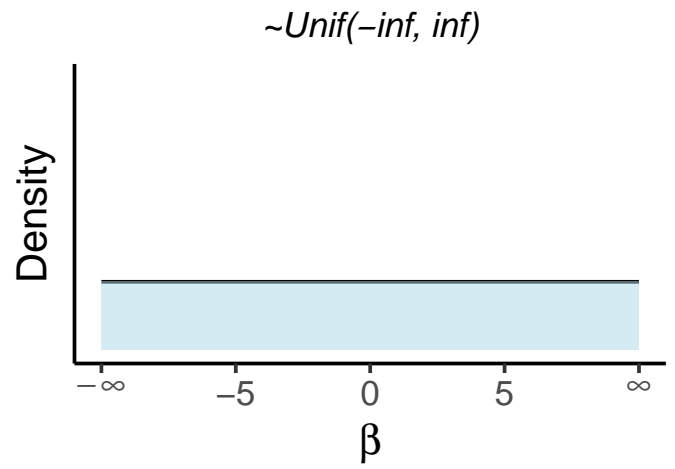

Intercept prior

$$
\sim T-\operatorname{student}(d f=3, m u=2, \text { scale }=10 \text { ) }
$$

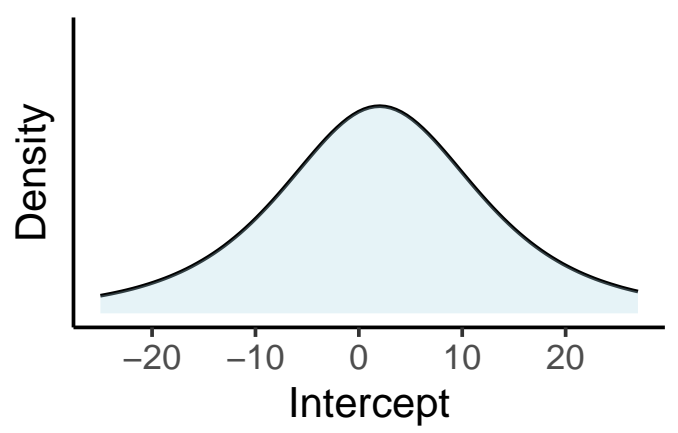

Random effect prior

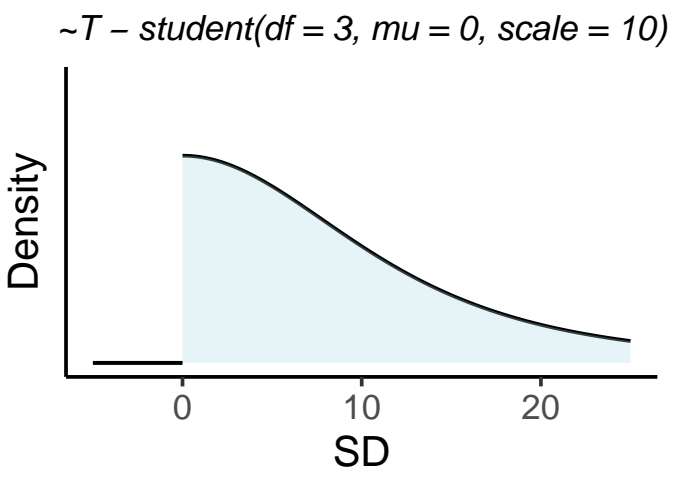

Random effect prior

$\sim \operatorname{Gamma}(0.01,0.01)$

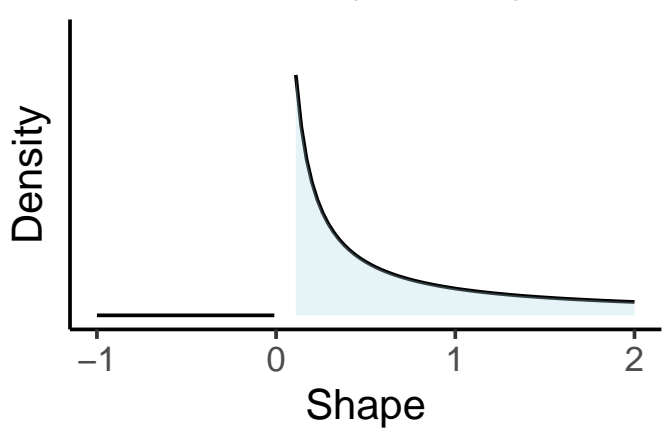

Figure 5: Parameter prior distributions 
Remember that by default, the population-level intercept is estimated separately and not as part of population-level parameter vector b. Moreover, to increase sampling efficiency, the population-level design matrix X is centered around its column means X_means. Thus, intercept prior is defined on the centered design matrix and not on the real intercept.

Below we report the default prior settings of brms for m.0. For the other models the settings are the same just with more $\beta$ parameters.

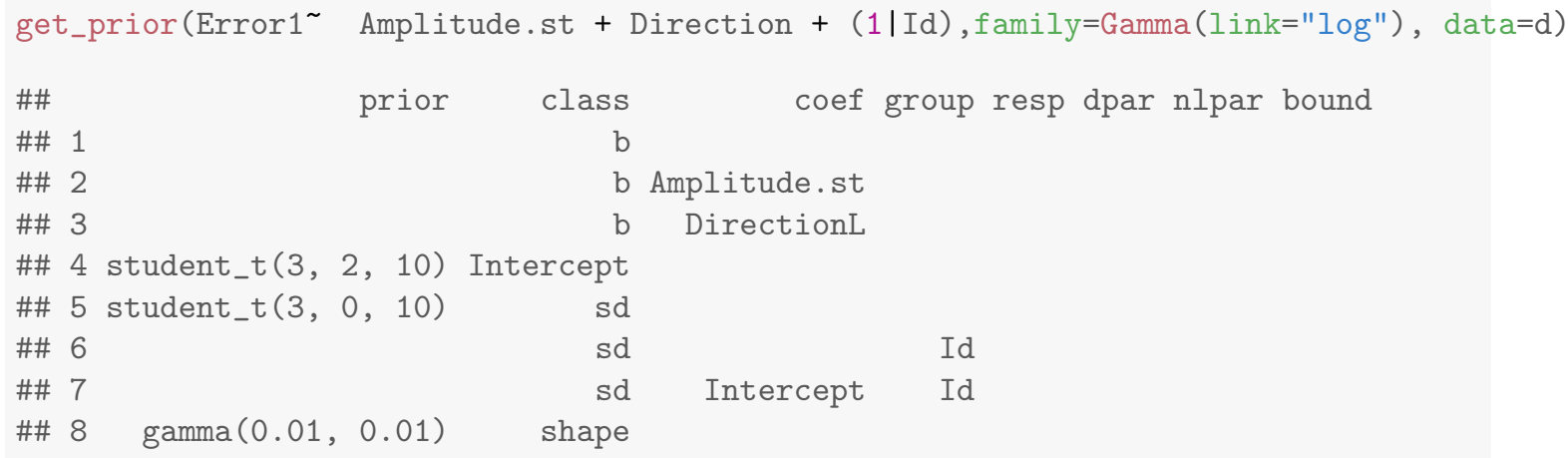

\subsection{Models Estimation}

Each model was estimated using 6 independent chains of 8,000 iterations with a "warm-up" period of 2,000 iterations, resulting in 36,000 usable samples.

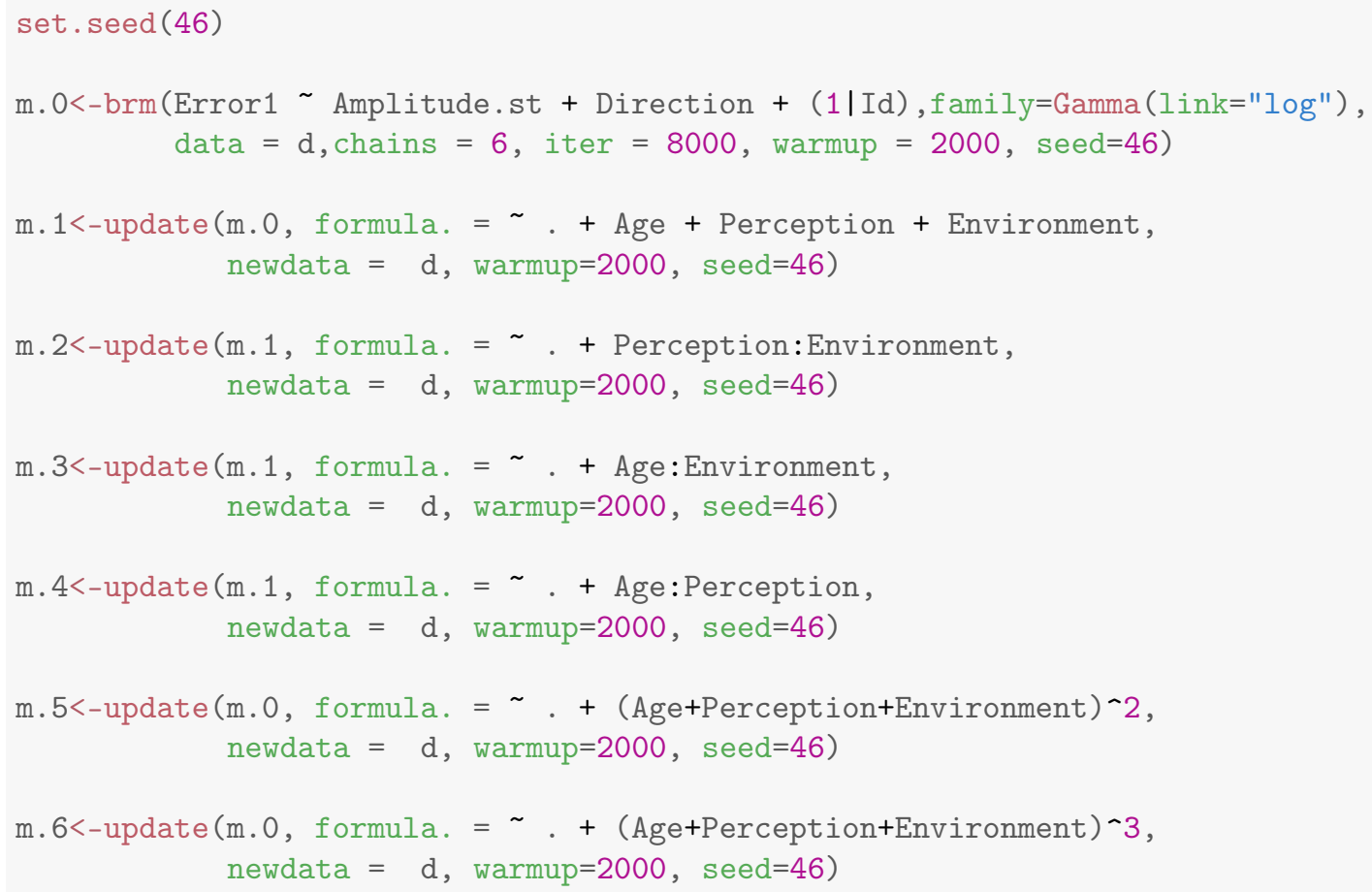

Convergence was evaluated via visual inspection of the trace plots (i.e. sampling chains) and Rhat diagnostic criteria (Gelman \& Rubin, 1992). All tested models showed satisfactory convergence with all $\mathrm{R}$-hat $\leq 1.00102$, where values close to 1 indicate convergence, and no R-hat exceeding the 1.100 proposed threshold for convergence (Gelman et al., 2013). R-hat values and trace plots are reported separetly for each model in the next sections. 


\subsection{1 m. 0}

In 6 the trace plots of the parameters in model m. 0 are presented. In 6 R-hat values are reported.
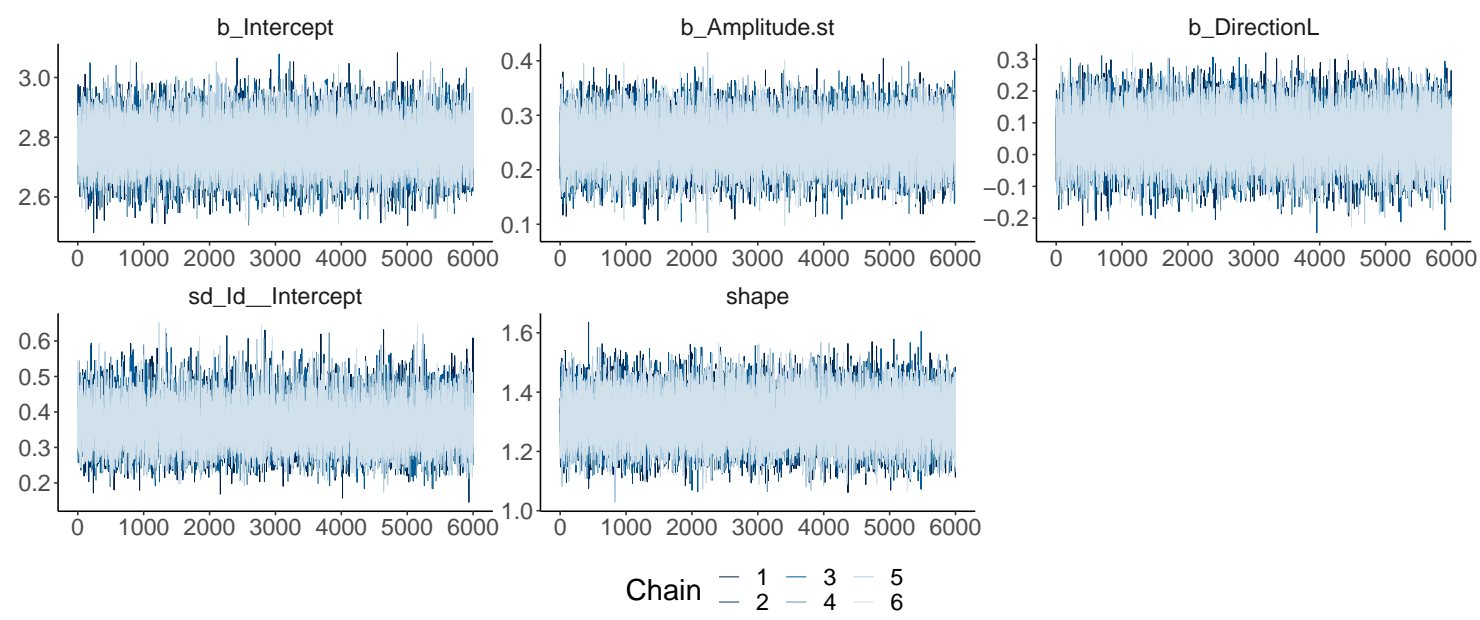

Figure 6: Parameter trace plots m.0. We only represent the iterations after the warm-up.

Table 6: R-hat values model m.0

\begin{tabular}{cc}
\hline Parameter & R-hat \\
\hline Intercept & \\
b_Intercept & 1.0000703 \\
Main Effects & \\
b_Amplitude.st & 0.9999496 \\
b_DirectionL & 0.9999334 \\
SD and Shape & \\
sd_Id_Intercept & 1.0002632 \\
shape & 0.9998980 \\
\hline
\end{tabular}




\subsection{2 m. 1}

In 7 the trace plots of the parameters in model $\mathrm{m} .1$ are presented. In $7 \mathrm{R}$-hat values are reported.
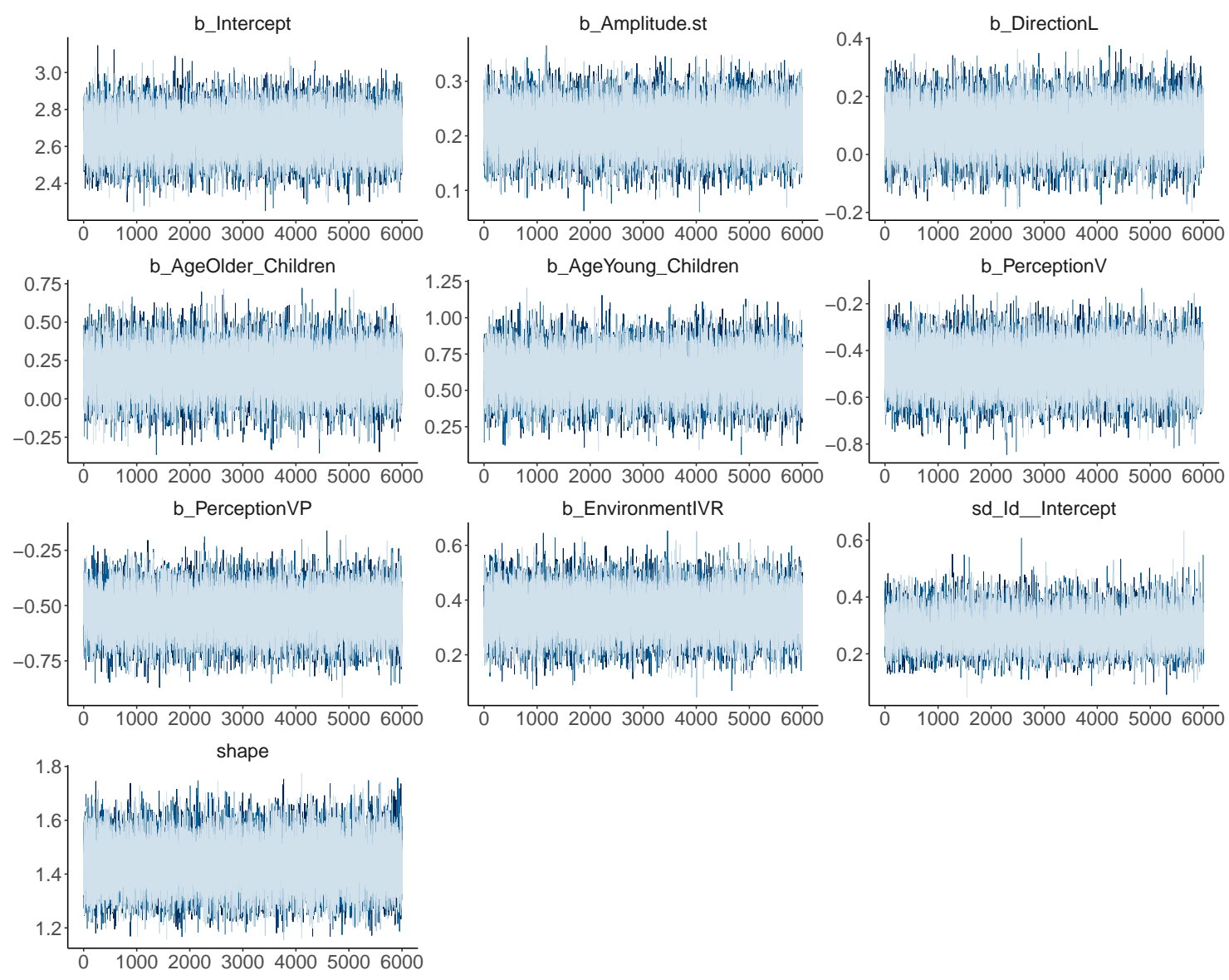

$$
\text { Chain }-1-3-5
$$

Figure 7: Parameter trace plots m.1. We only represent the iterations after the warm-up. 
Table 7: R-hat values model m.1

\begin{tabular}{rc}
\hline Parameter & R-hat \\
\hline Intercept & \\
b_Intercept & 1.0000278 \\
Main Effects & \\
b_Amplitude.st & 0.9999213 \\
b_DirectionL & 0.9998864 \\
b_AgeOlder_Children & 1.0000970 \\
b_AgeYoung_Children & 0.9999157 \\
b_PerceptionV & 0.9999481 \\
b_PerceptionVP & 0.9999765 \\
b_EnvironmentIVR & 1.0000876 \\
SD and Shape & \\
sd_Id__Intercept & 1.0005022 \\
shape & 1.0000356 \\
\hline
\end{tabular}




\subsection{3 m. 2}

In 8 the trace plots of the parameters in model m. 2 are presented. In 8 R-hat values are reported.
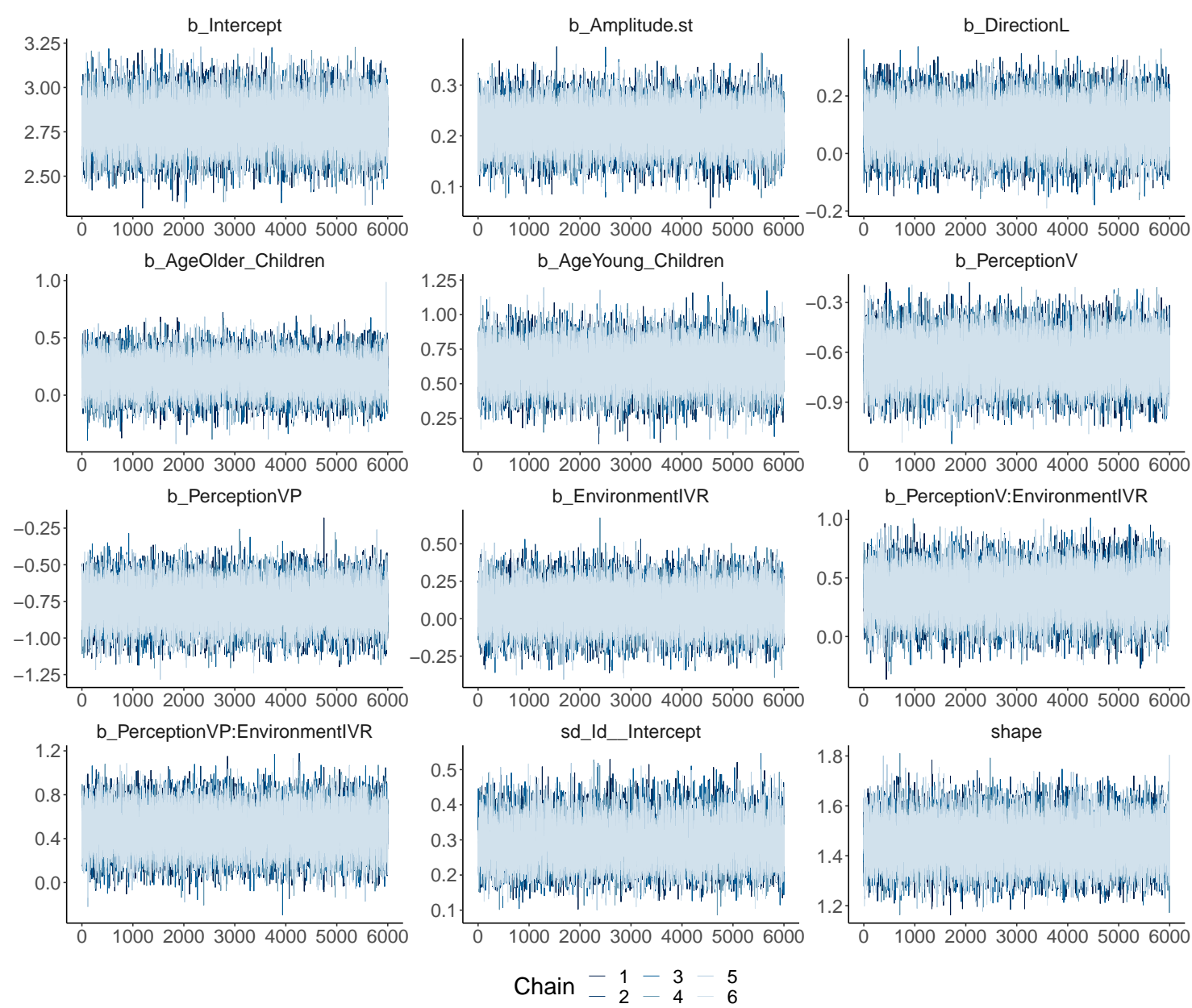

Figure 8: Parameter trace plots m.2. We only represent the iterations after the warm-up. 
Table 8: R-hat values model m.2

\begin{tabular}{lrr}
\hline & Parameter & R-hat \\
\hline Intercept & b_Intercept & 0.9999611 \\
Main Effects & & \\
& b_Amplitude.st & 0.9999866 \\
& b_DirectionL & 0.9999106 \\
b_AgeOlder_Children & 1.0000100 \\
b_AgeYoung_Children & 1.0000832 \\
& b_PerceptionV & 0.9999024 \\
b_PerceptionVP & 0.9999252 \\
b_EnvironmentIVR & 0.9999110 \\
Interaction Effects & \\
b_PerceptionV:EnvironmentIVR & 1.0000029 \\
b_PerceptionVP:EnvironmentIVR & 0.9999393 \\
SD and Shape & \\
sd_Id__Intercept & 1.0001767 \\
shape & 0.9999588 \\
\hline
\end{tabular}




\subsection{4 m.3}

In 9 the trace plots of the parameters in model m. 3 are presented. In 9 R-hat values are reported.
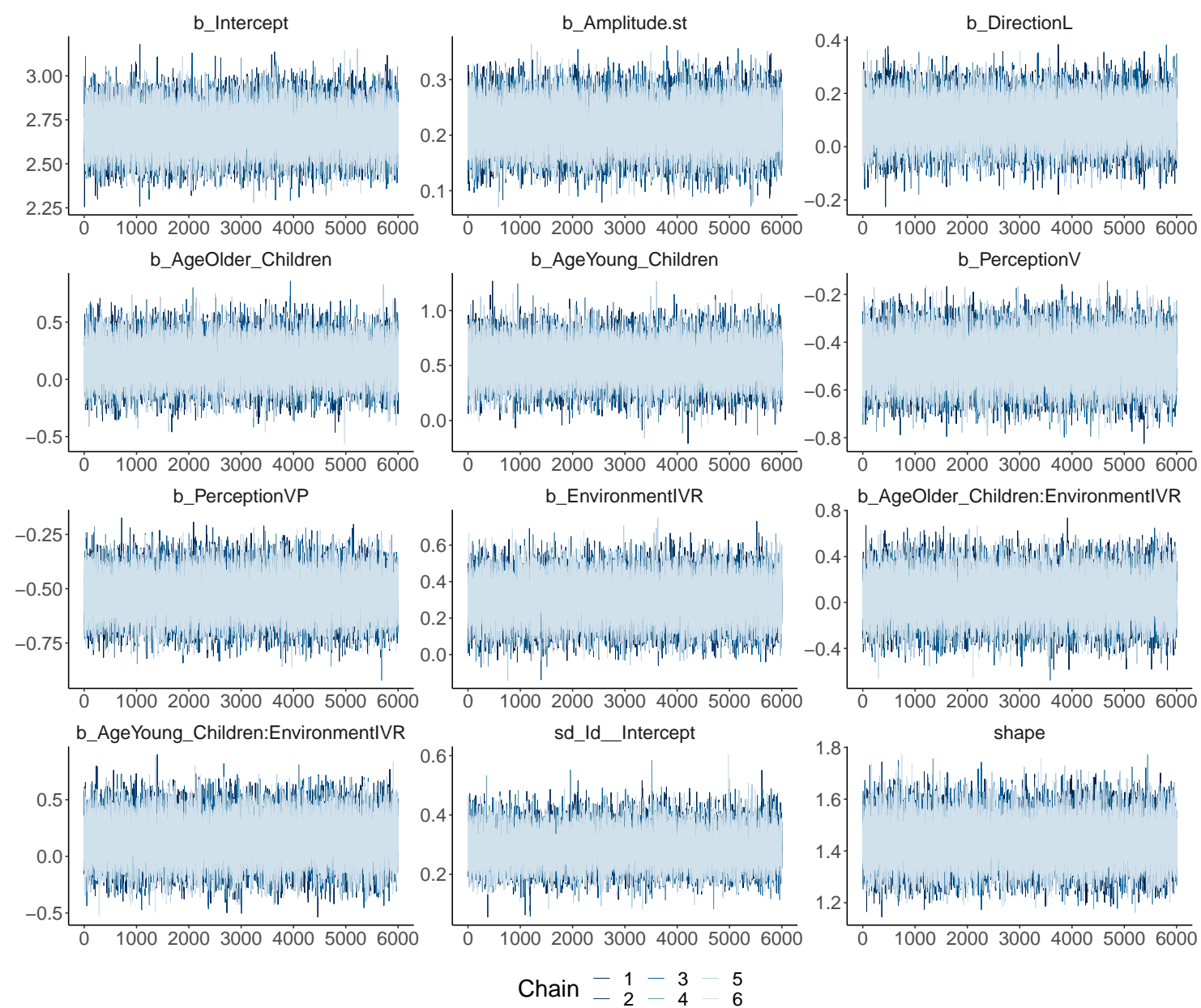

Figure 9: Parameter trace plots m.3. We only represent the iterations after the warm-up. 
Table 9: R-hat values model m.3

\begin{tabular}{lrr}
\hline & Parameter & R-hat \\
\hline Intercept & b_Intercept & 1.0000509 \\
Main Effects & b_Amplitude.st & 0.9998889 \\
& b_DirectionL & 0.9999307 \\
& b_AgeOlder_Children & 0.9999849 \\
& b_AgeYoung_Children & 1.0000911 \\
& b_PerceptionV & 0.9999024 \\
& b_PerceptionVP & 0.9998772 \\
b_EnvironmentIVR & 0.9999883 \\
b_AgeOlder_Children:EnvironmentIVR & 0.9999206 \\
b_AgeYoung_Children:EnvironmentIVR & 0.9999421 \\
SD and Shape & sd_Id_Intercept & 1.0001182 \\
& shape & 0.9999171 \\
\hline
\end{tabular}




\subsection{5 m.4}

In 10 the trace plots of the parameters in model m. 4 are presented. In $10 \mathrm{R}$-hat values are reported.
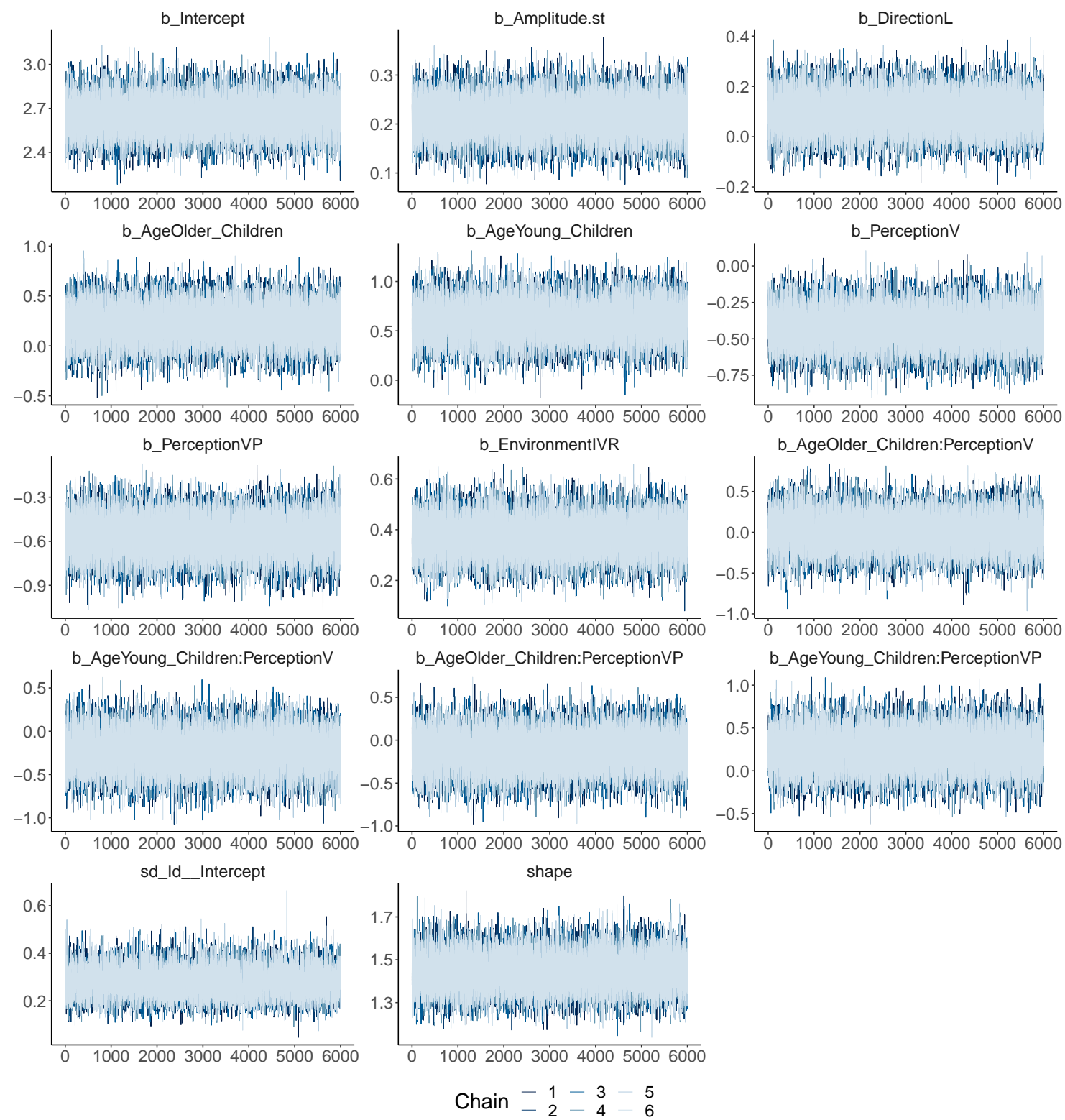

Figure 10: Parameter trace plots m.4. We only represent the iterations after the warm-up. 
Table 10: R-hat values model m.4

\begin{tabular}{lrr}
\hline & Parameter & R-hat \\
\hline Intercept & b_Intercept & 1.0002654 \\
Main Effects & b_Amplitude.st & 0.9999213 \\
& b_DirectionL & 0.9999336 \\
& b_AgeOlder_Children & 1.0001077 \\
b_AgeYoung_Children & 1.0002796 \\
& b_PerceptionV & 0.9999924 \\
b_PerceptionVP & 1.0000110 \\
b_EnvironmentIVR & 0.9999741 \\
Interaction Effects & \\
b_AgeOlder_Children:PerceptionV & 1.0000142 \\
b_AgeYoung_Children:PerceptionV & 0.9999901 \\
b_AgeOlder_Children:PerceptionVP & 1.0000328 \\
b_AgeYoung_Children:PerceptionVP & 1.0000558 \\
SD and Shape & & \\
sd_Id_Intercept & 1.0003760 \\
& shape & 0.9998865 \\
\hline
\end{tabular}




\subsection{6 m. 5}

In 11 the trace plots of the parameters in model m. 5 are presented. In 11 R-hat values are reported.
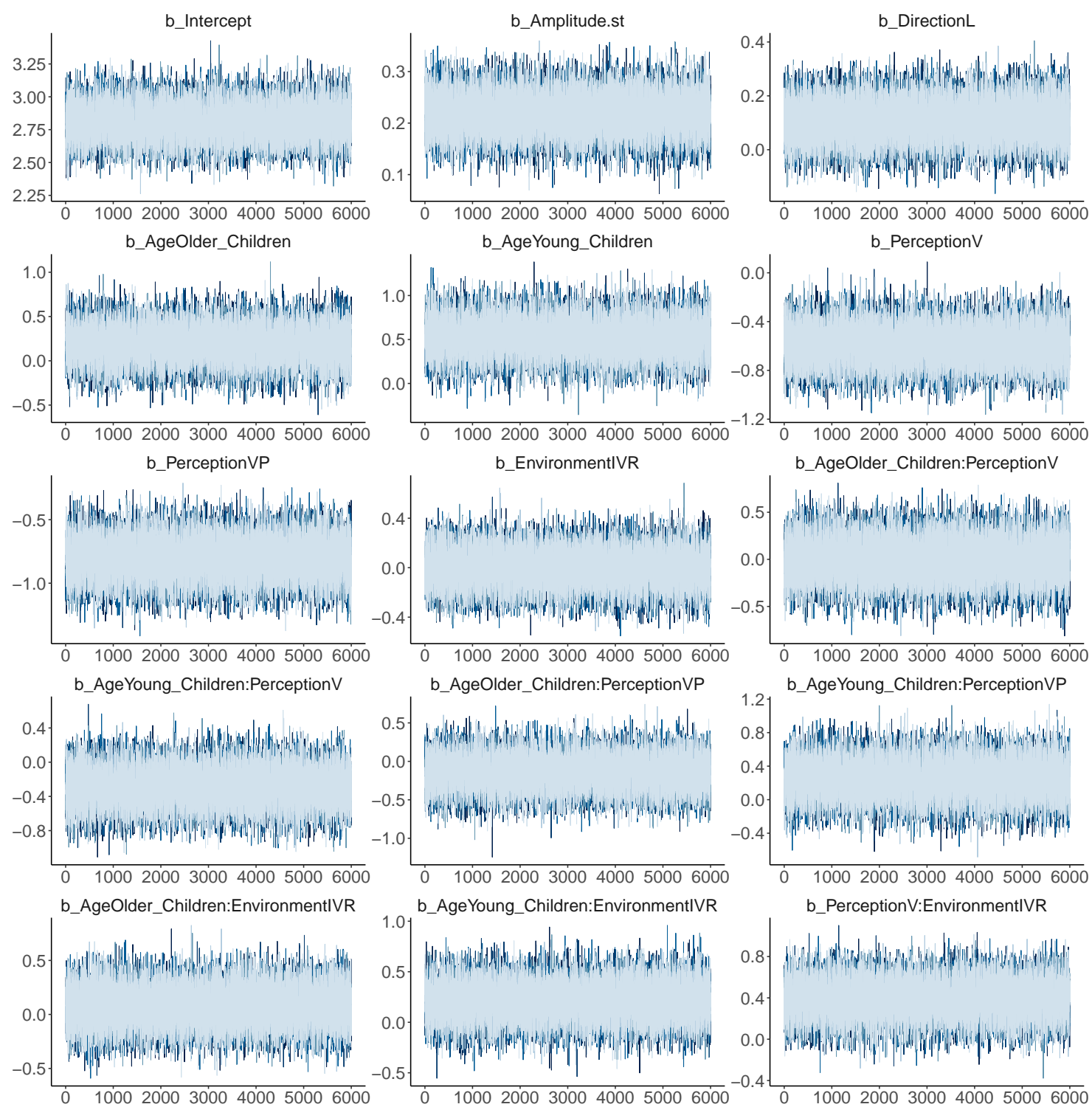

b_PerceptionV:EnvironmentIVR
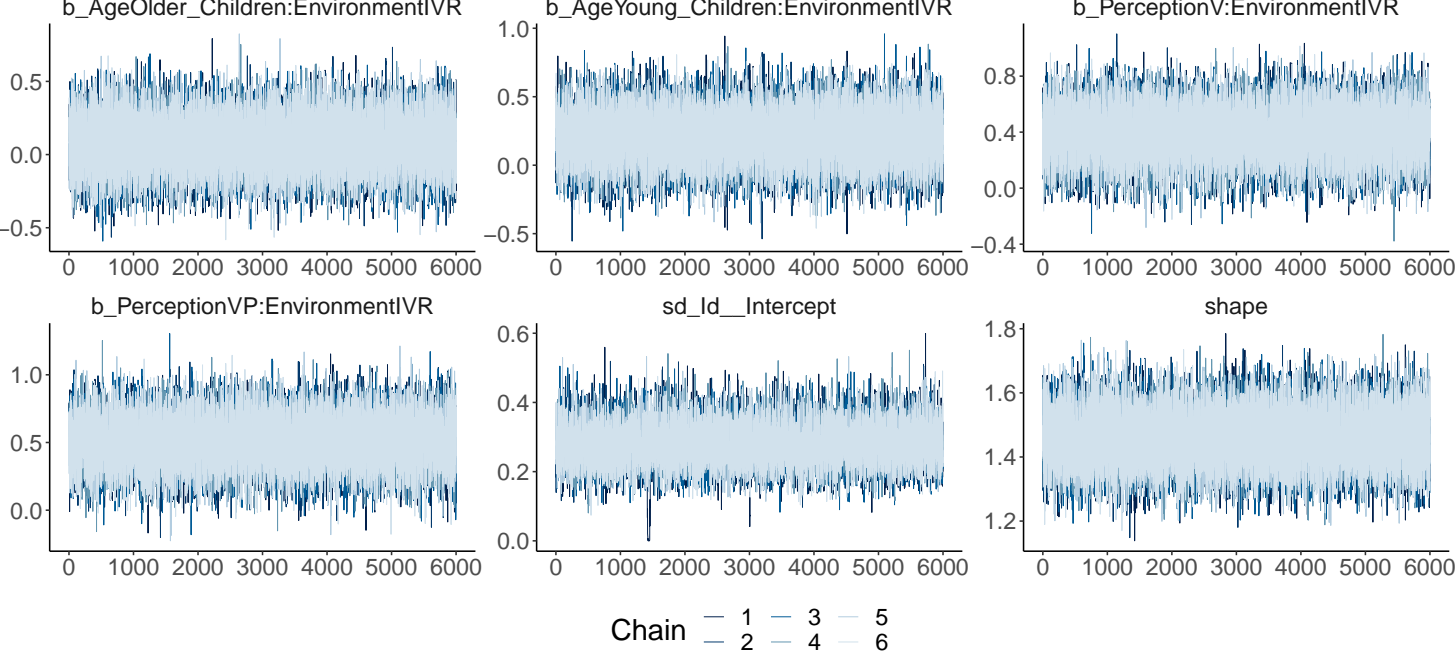

Figure 11: Parameter trace plots m.5. We only represent the iterations after the warm-up. 
Table 11: R-hat values model m.5

\begin{tabular}{|c|c|}
\hline Parameter & R-hat \\
\hline \multicolumn{2}{|l|}{ Intercept } \\
\hline b_Intercept & 1.0002476 \\
\hline \multicolumn{2}{|l|}{ Main Effects } \\
\hline b_Amplitude.st & 0.9999448 \\
\hline$\overline{\mathrm{b}} \_$DirectionL & 1.0000320 \\
\hline b_AgeOlder_Children & 1.0002600 \\
\hline b_AgeYoung_Children & 1.0003221 \\
\hline b PerceptionV & 1.0000061 \\
\hline b_PerceptionVP & 1.0001615 \\
\hline b_EnvironmentIVR & 1.0000616 \\
\hline \multicolumn{2}{|l|}{ Interaction Effects } \\
\hline b AgeOlder Children:PerceptionV & 1.0000133 \\
\hline b_AgeYoung_Children:PerceptionV & 1.0000105 \\
\hline b_AgeOlder_Children:PerceptionVP & 1.0000587 \\
\hline b_AgeYoung_Children:PerceptionVP & 1.0001262 \\
\hline b_AgeOlder_Children:EnvironmentIVR & 1.0000978 \\
\hline b_AgeYoung_Children:EnvironmentIVR & 1.0001040 \\
\hline b_PerceptionV:EnvironmentIVR & 0.9999806 \\
\hline b_PerceptionVP:EnvironmentIVR & 1.0000506 \\
\hline \multicolumn{2}{|l|}{ SD and Shape } \\
\hline sd_Id__Intercept & 1.0006684 \\
\hline-- shape & 0.9999478 \\
\hline
\end{tabular}




\subsection{7 m.6}

In 12 the trace plots of the parameters in model m. 6 are presented. In $12 \mathrm{R}$-hat values are reported.
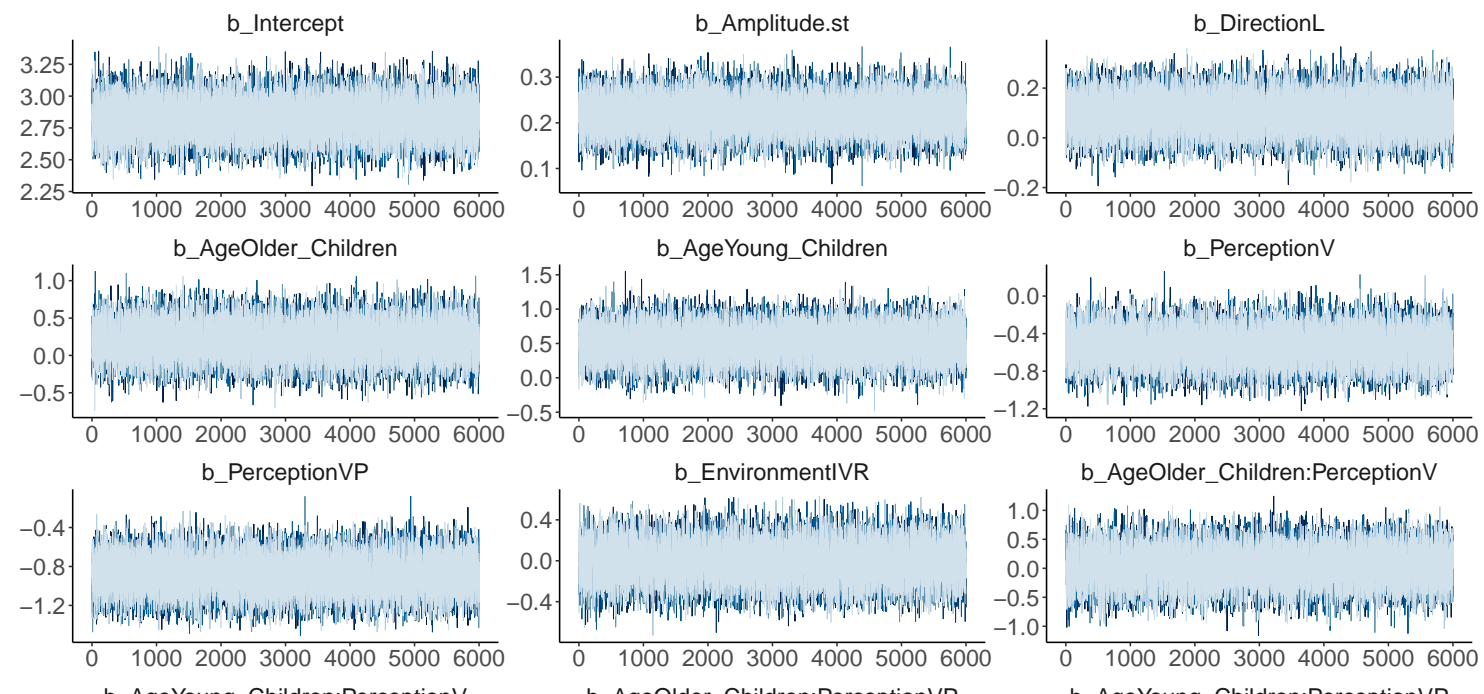

b_EnvironmentIVR

b_AgeOlder_Children:PerceptionV
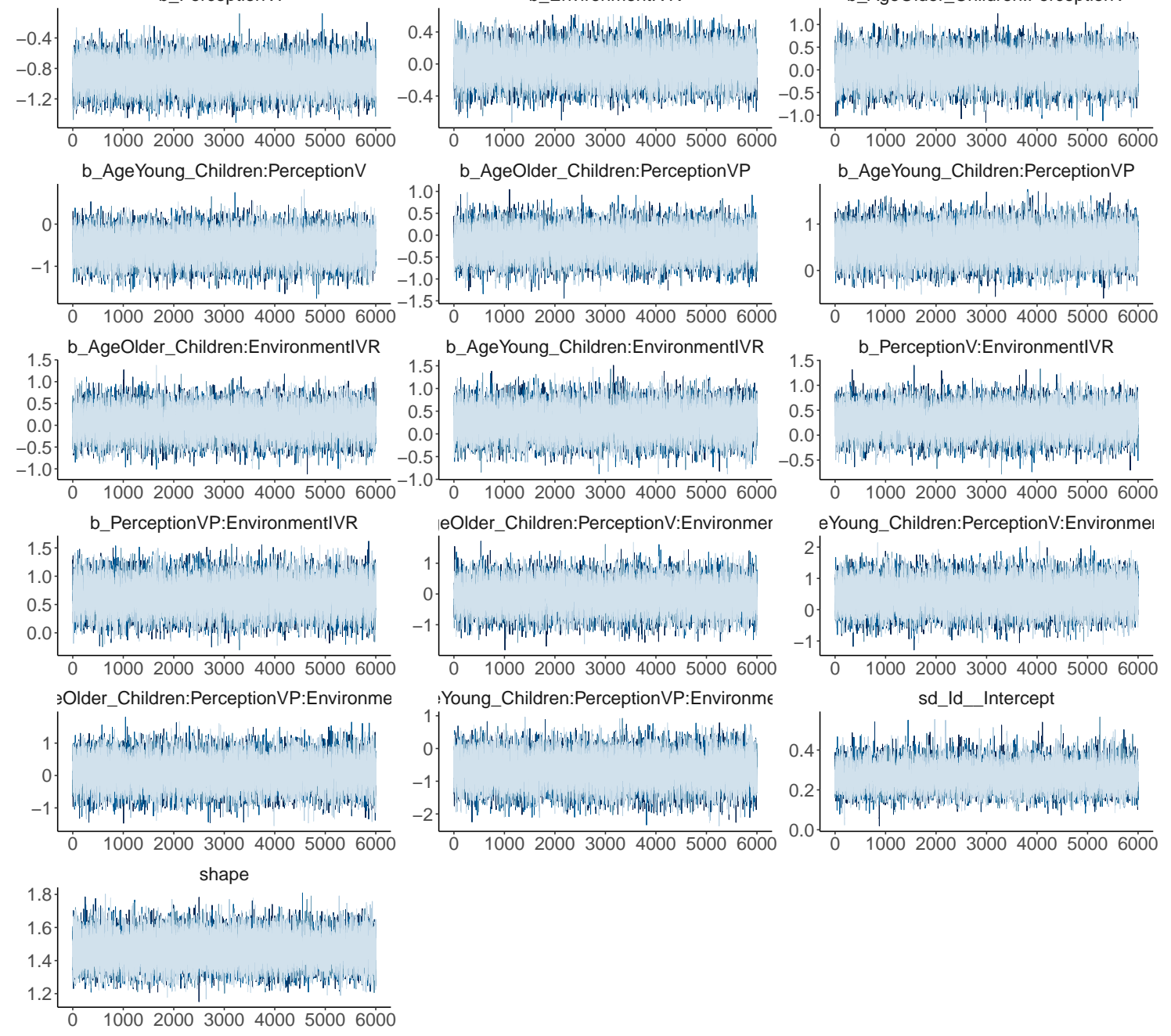

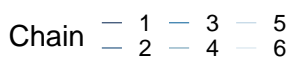

Figure 12: Parameter trace plots m.6. We only represent the iterations after the warm-up. 
Table 12: R-hat values model m. 6

\begin{tabular}{lrc}
\hline & Parameter & R-hat \\
\hline Intercept & b_Intercept & 1.0002811 \\
Main Effects & b_Amplitude.st & 0.9999474 \\
b_DirectionL & 0.9998861 \\
& b_AgeOlder_Children & 1.0003139 \\
b_AgeYoung_Children & 1.0002440 \\
b_PerceptionV & 1.0000941 \\
b_PerceptionVP & 1.0003398 \\
b_EnvironmentIVR & 1.0004737
\end{tabular}

Interaction Effects

b AgeOlder Children:PerceptionV 1.0001320

b_AgeYoung_Children:PerceptionV 1.0000189

b_AgeOlder_Children:PerceptionVP 1.0004903

b_AgeYoung_Children:PerceptionVP 1.0001956

b_AgeOlder_Children:EnvironmentIVR 1.0002921

b_AgeYoung_Children:EnvironmentIVR 1.0002693

b_PerceptionV:EnvironmentIVR 1.0000812

b_PerceptionVP:EnvironmentIVR 1.0004311

Three-ways Interactions

b_AgeOlder_Children:PerceptionV:EnvironmentIVR 1.0001678

b_AgeYoung_Children:PerceptionV:EnvironmentIVR 1.0000836

b_AgeOlder_Children:PerceptionVP:EnvironmentIVR 1.0003083

b_AgeYoung_Children:PerceptionVP:EnvironmentIVR 1.0001604

SD and Shape

$\begin{array}{rr}\text { sd_Id__Intercept } & 0.9999680 \\ \text { shape } & 0.9998776\end{array}$




\subsection{Model Comparison}

The Watanabe-Akaike Information Criterion (WAIC; Gelman, Hwang, \& Vehtari, 2014 Vehtari, Gelman, \& Gabry, 2017) is used as information criteria to select the most plausible model among the tested models, given the data. WAIC is the corresponding Bayesian version of the commonly used Akaike information criterion (AIC; Akaike, 1973). In line with the Bayesian approach, WAIC has the desirable property of averaging over the posterior distribution rather than conditioning on a point estimate.

Moreover, WAIC-weights for the set of models are computed to present the probability of each model to make the best predictions on new data, conditional on the set of models considered (McElreath, 2016). With this method models are compared using a continuous informative measure of evidence, rather than a dichotomous decision, to avoid premature conclusions in this explorative scenario.

First we compute the WAIC for each model.

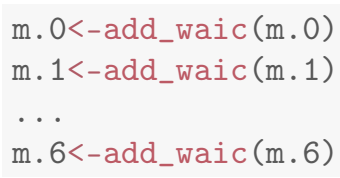

Then we compute the relative WAIC weights.

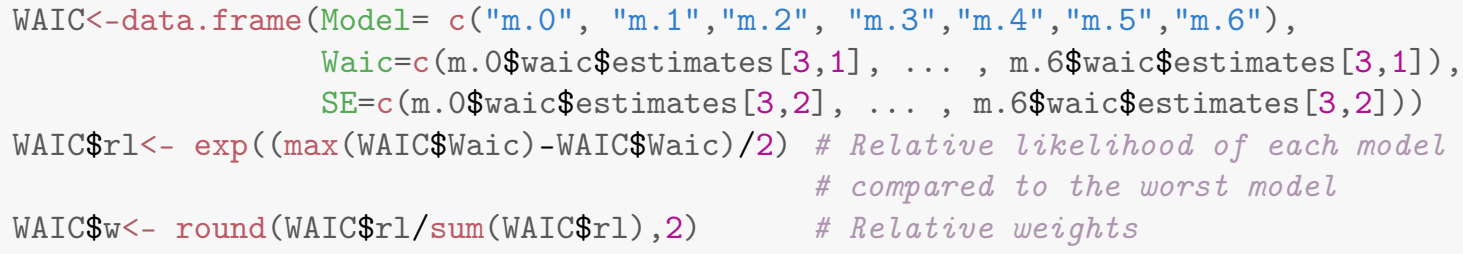

WAIC values and WAIC weights are reported in 13

Table 13: WAIC model comparison

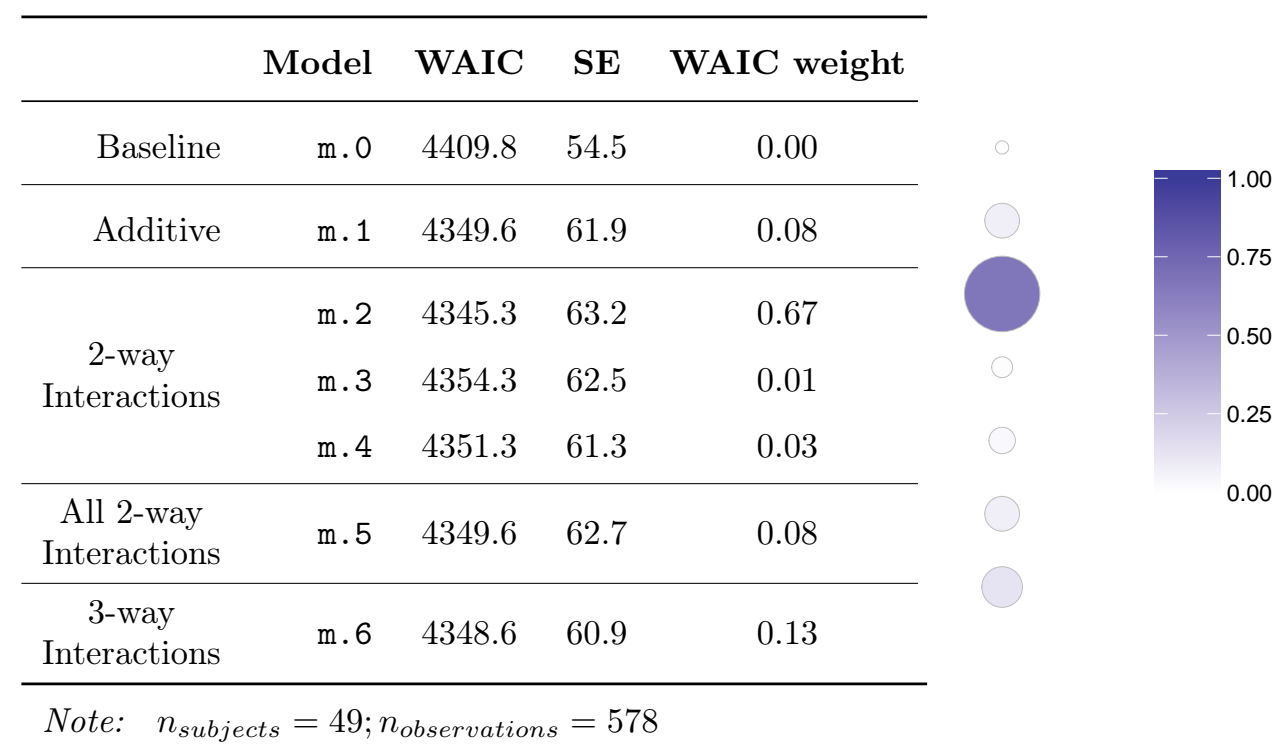

Results indicate that m. 2 is the most plausible model that has generated the observed data, having the lower WAIC value $(\mathrm{WAIC}=4345.3)$ and a probability of being the best of 0.67 . 
Compared with $\mathrm{m} .6$, the second-most plausible model with a probability of $0.13, \mathrm{~m} .2$ is 5.14 times more probable. 


\section{Description of Model m.2}

In this section we will first report the estimated parameter of the model m.2. Main effects and interaction effects are considered using planned comparison and graphical representations of the predicted values by the model. Moreover, effects sizes are presented. Finally, we will evaluate the model fit considering $R^{2}$ value and model Posterior Predictive check.

\subsection{Model Parameters}

We recall that model m.2 considers the fixed effects of Age, Amplitude.st, Direction, the interaction between Perception and Environment, and the random-effect of Id.

In 14 95\% Bayesian Credible Intervals (BCIs) of the parameter posterior distributions are presented. $95 \%$ BCIs represent the range of the $95 \%$ most credible parameter values given the prior distribution and the observed data.

Table 14: Estimated parameters of model m.2

\begin{tabular}{|c|c|c|c|c|c|c|c|}
\hline & \multicolumn{3}{|c|}{ Parameters } & \multicolumn{2}{|c|}{$95 \% \mathrm{BCI}$} & \multicolumn{2}{|c|}{ Eff.Sample } \\
\hline & Name & Estimate & Est.Error & Lower & Upper & Bulk & Tail \\
\hline \multicolumn{8}{|c|}{ Random Effects } \\
\hline SD & ID Intercept & 0.29 & 0.05 & 0.19 & 0.41 & 15425 & 23447 \\
\hline \multicolumn{8}{|c|}{ Fixed Effect } \\
\hline & Intercept & 2.79 & 0.12 & 2.57 & 3.03 & 30749 & 29866 \\
\hline & Amplitude.st & 0.22 & 0.04 & 0.14 & 0.29 & 76919 & 28513 \\
\hline & Direction (left) & 0.10 & 0.07 & -0.04 & 0.23 & 79506 & 27632 \\
\hline & Environment (IVR) & 0.08 & 0.12 & -0.16 & 0.32 & 29652 & 28643 \\
\hline \multirow{2}{*}{ Age } & Older Children & 0.18 & 0.13 & -0.09 & 0.44 & 28789 & 27196 \\
\hline & Young Children & 0.62 & 0.13 & 0.36 & 0.89 & 29571 & 28560 \\
\hline \multirow{2}{*}{ Perception } & Vision & -0.64 & 0.12 & -0.89 & -0.40 & 35596 & 30037 \\
\hline & Vision + Proprioception & -0.77 & 0.12 & -1.01 & -0.53 & 34851 & 30305 \\
\hline \multirow[b]{2}{*}{ Interaction } & Vision : IVR & 0.36 & 0.17 & 0.02 & 0.70 & 32305 & 29312 \\
\hline & $\begin{array}{r}\text { Vision }+ \text { Proprioception : } \\
\text { IVR }\end{array}$ & 0.48 & 0.17 & 0.14 & 0.82 & 31344 & 29433 \\
\hline \multicolumn{8}{|c|}{ Family Specific Parameter } \\
\hline & shape & 1.45 & 0.08 & 1.30 & 1.61 & 64978 & 26539 \\
\hline
\end{tabular}

Given the results, Self-turn error is moderated by Amplitude.st, Age and by the interaction between Perception and Environment. On the contrary, the Direction of rotations seems to have no effect on the subjects' performance $(\beta=0.1 ; 95 \% B C I=-0.04 ; 0.23)$.

In order to interpret the results, we used the posterior parameter distributions to compute, in the original scale, the predicted distribution of Self-turn error means for the condition we are interested in, marginalizing for the other variables.

For example, to obtain the distribution of Self-turn error means for Adults in the Proprioception and Reality condition, which are the baseline model categories, we marginalize (i.e. compute the average score) over the variable direction. 


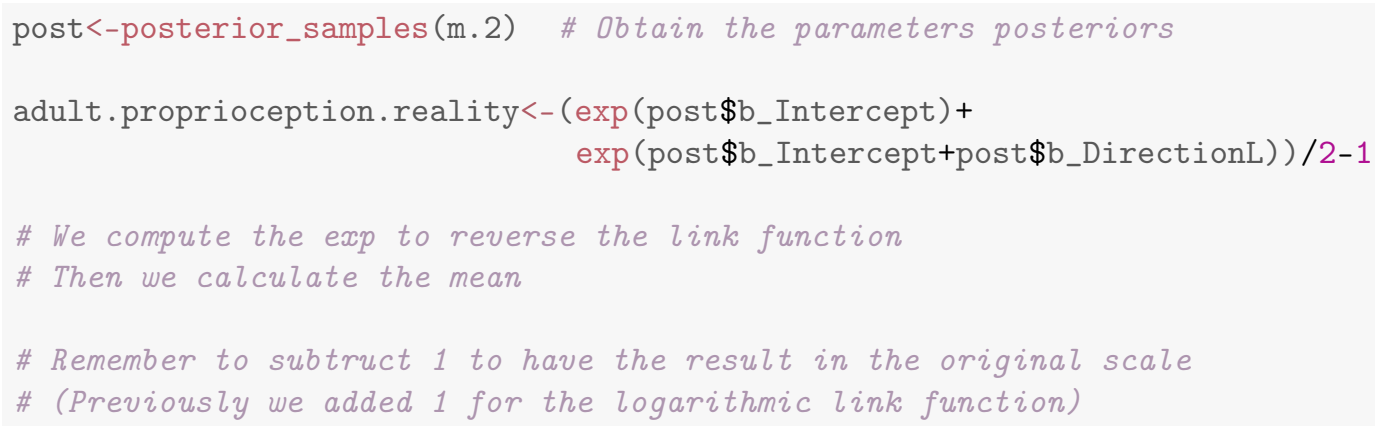

\subsubsection{Amplitude}

Self-turn error is moderated by Amplitude.st $(\beta=0.22 ; 95 \% B C I=0.14 ; 0.29)$, for which increasing levels of task difficulty are associated with a worse performance ( 13$)$.

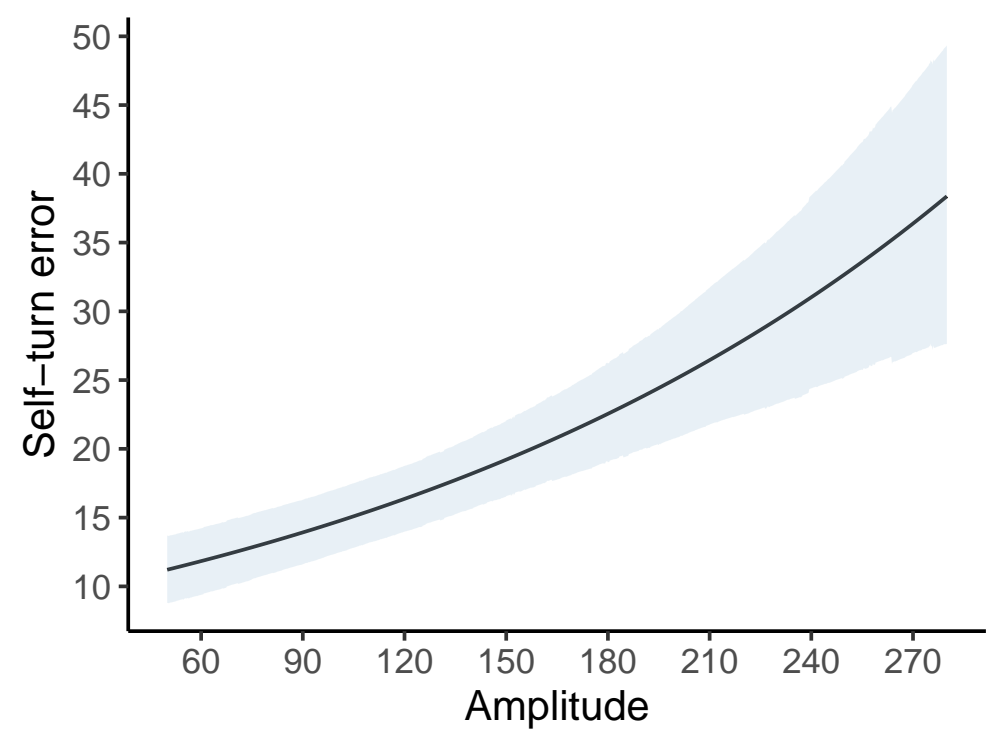

Figure 13: Predicted mean of Self-turn error according to Amplitude.st $\left(n_{\text {subjects }}=49\right.$; $\left.n_{\text {observations }}=578\right)$. The line represents the mean value. The shaded area represents the $95 \%$ $\mathrm{BCI}$ values.

\subsubsection{Age}

To evaluate the role of Age, the distributions of predicted mean values for the three groups are presented in 14.

The predicted mean error for Adults is 12.8 degrees $(95 \% B C I=10.6 ; 15.1)$, for Older Children 15.5 degrees $(95 \% B C I=12.1 ; 19.2)$ and for Young Children is 24.8 degrees $(95 \% B C I=$ $19.3 ; 30.8)$. Values are reported in 15 together with Bayesian pairwise comparisons (i.e. predicted score differences between groups). 


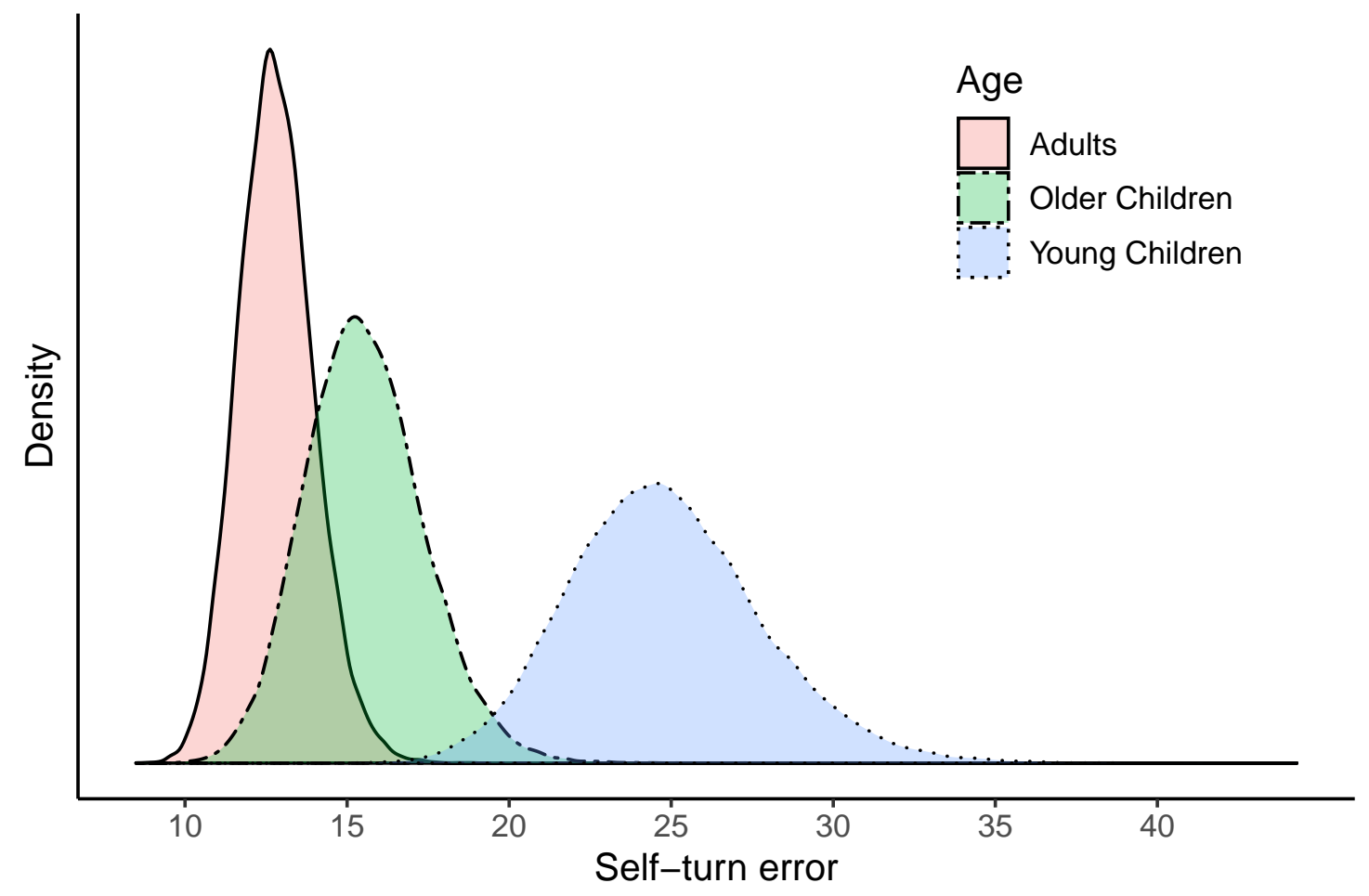

Figure 14: Distributions of the predicted means of Self-turn error according to Age $\left(n_{\text {subjects }}=49\right.$; $\left.n_{\text {observations }}=578\right)$.

Overall, Young Children are expected to make more Self-turn error than Adults $(95 \% B C I=$ $6.3 ; 18.2)$ and also more than Older Children $(95 \% B C I=2.8 ; 16)$. On the contrary, we cannot state that Older Children are expected to make more Self-turn error because the 95\% BCI of the difference includes the value zero $(95 \% B C I=-1.4 ; 6.9)$.

Table 15: Predicted means and differences of Self-turn error according to age.

\begin{tabular}{|c|c|c|c|}
\hline \multicolumn{2}{|l|}{ Mean } & \multicolumn{2}{|c|}{$95 \%$ BCI } \\
\hline & Estimate & Lower & Upper \\
\hline \multicolumn{4}{|l|}{ Group } \\
\hline Adults & 12.8 & 10.6 & 15.1 \\
\hline Older Children & 15.5 & 12.1 & 19.2 \\
\hline Young Children & 24.8 & 19.3 & 30.8 \\
\hline \multicolumn{4}{|l|}{ Comparisons } \\
\hline Young Children - Adults & 12.0 & 6.3 & 18.2 \\
\hline Young - Older Children & 9.3 & 2.8 & 16.0 \\
\hline Older Children - Adults & 2.7 & -1.4 & 6.9 \\
\hline
\end{tabular}




\subsubsection{Interaction between Perception and Environment}

To interpret the interaction between Perception and Environment conditions, the distributions of predicted mean values for all six conditions are presented in 15 .

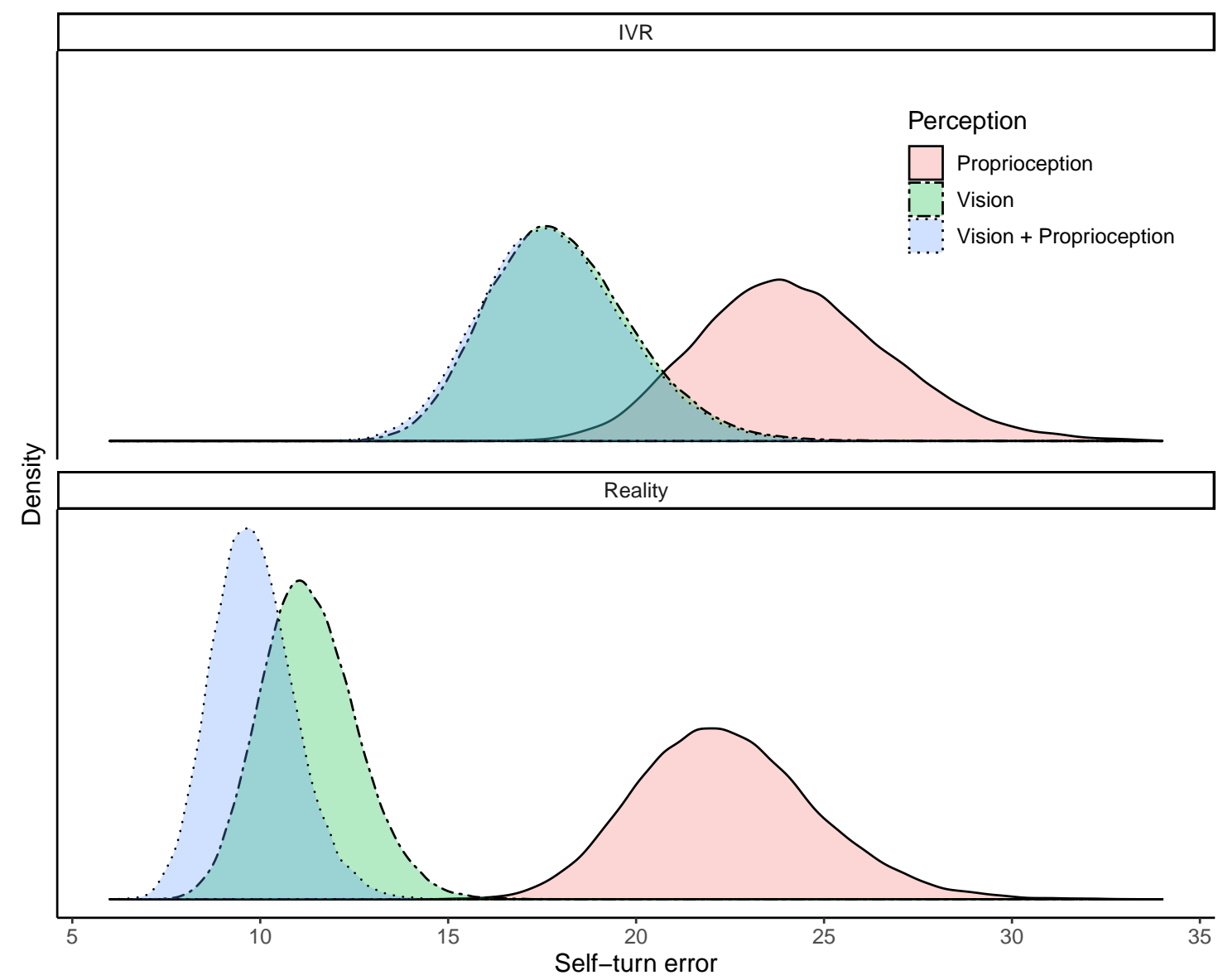

Figure 15: Distributions of the predicted means of Self-turn error according to the experimental conditions $\left(n_{\text {subjects }}=49 ; n_{\text {observations }}=578\right)$.

In the Reality Environment, the predicted mean error for Proprioception is 22.4 degrees $(95 \% B C I=18 ; 27.2)$, for Vision 11.3 degrees $(95 \% B C I=9 ; 13.9)$ and for Vision + Proprioception is 9.8 degrees $(95 \% B C I=7.8 ; 12)$. In the IVR Environment, the predicted mean error for Proprioception is 24.3 degrees $(95 \% B C I=19.3 ; 29.2)$, for Vision 18 degrees $(95 \% B C I=14.4 ; 21.8)$ and for Vision + Proprioception is 17.8 degrees $(95 \% B C I=14.2 ; 21.7)$. Values are reported in 16 together with Bayesian pairwise comparisons (i.e. predicted score differences between conditions). 
Table 16: Predicted means and differences of Self-turn error according experimental conditions.

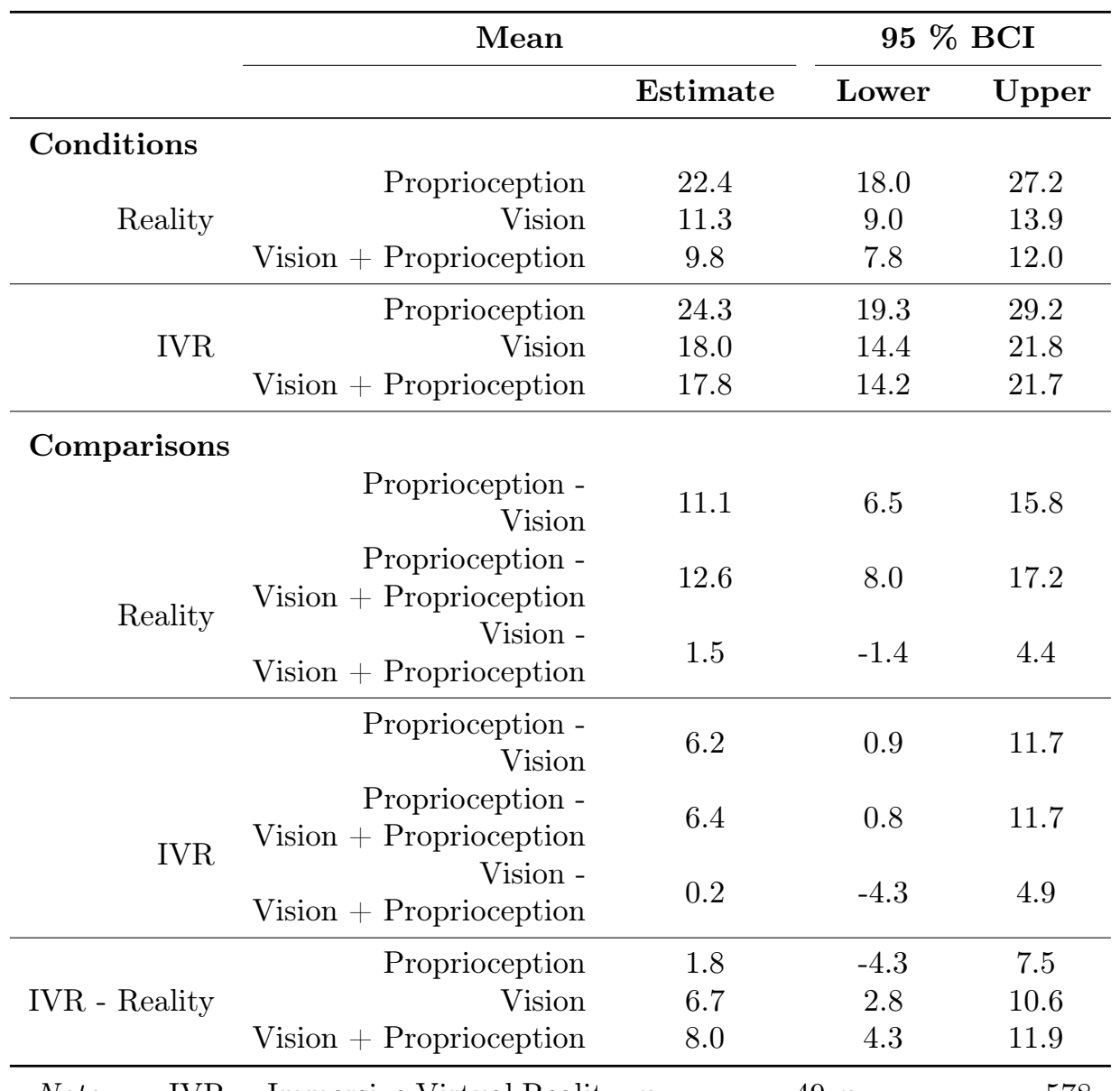

Note: $\quad$ IVR $=$ Immersive Virtual Reality. $n_{\text {subjects }}=49 ; n_{\text {observations }}=578$

Results showed that, in Reality and IVR Environment, subjects are expected to make more Self-turn error when they rely only on Proprioception than when they can use only Vision (Reality: $95 \% B C I=6.5 ; 15.8$; IVR: $95 \% B C I=0.9 ; 11.7$ ) or Vision + Proprioception (Reality: $95 \% B C I=$ 8; 17.2; IVR: $95 \% B C I=0.8 ; 11.7)$. On the contrary, there is no difference between the use of Vision and Vision + Proprioception (Reality: $95 \% B C I=-1.4 ; 4.4$; IVR: $95 \% B C I=-4.3 ; 4.9$ ). Moreover, compariong IVR to Reality Environment, Self-turn errors increase when subjects rely only on Vision $(95 \% B C I=2.8 ; 10.6)$ or on Vision + Proprioception $(95 \% B C I=4.3 ; 11.9)$, but subjects are not expected to make the more errors when they rely only on Proprioception $(95 \% B C I=-4.3 ; 7.5)$.

\section{$5.2 \quad$ Effect Sizes}

To quantify the differences between the various age groups or the different conditions, we expressed the effects as the ratio between the two scores of the comparison of interest (Table 17). 
Table 17: Effect size as the ratio of the scores of the different Age groups or experimental conditions.

\begin{tabular}{|c|c|c|c|c|}
\hline & \multicolumn{2}{|l|}{ Effect Size } & \multicolumn{2}{|c|}{$95 \%$ BCI } \\
\hline & & Estimate & Lower & Upper \\
\hline \multicolumn{5}{|l|}{ Age } \\
\hline & Young Children / Adults & 1.88 & 1.39 & 2.37 \\
\hline & Young / Older Children & 1.58 & 1.12 & 2.06 \\
\hline & Older Children / Adults & 1.20 & 0.90 & 1.53 \\
\hline \multicolumn{5}{|c|}{ Conditions } \\
\hline \multirow{3}{*}{ Reality } & Proprioception / Vision & 1.92 & 1.48 & 2.41 \\
\hline & $\begin{array}{r}\text { Proprioception / } \\
\text { Vision }+ \text { Proprioception }\end{array}$ & 2.18 & 1.68 & 2.73 \\
\hline & $\begin{array}{r}\text { Vision / } \\
\text { Vision }+ \text { Proprioception }\end{array}$ & 1.15 & 0.87 & 1.43 \\
\hline \multirow{3}{*}{ IVR } & Proprioception / Vision & 1.34 & 1.03 & 1.66 \\
\hline & $\begin{array}{r}\text { Proprioception / } \\
\text { Vision }+ \text { Proprioception }\end{array}$ & 1.35 & 1.03 & 1.68 \\
\hline & Vision + Proprioception & 1.02 & 0.78 & 1.26 \\
\hline \multirow{3}{*}{$\begin{array}{l}\text { IVR / } \\
\text { Reality }\end{array}$} & Proprioception & 1.09 & 0.83 & 1.34 \\
\hline & Vision & 1.56 & 1.19 & 1.94 \\
\hline & Vision + Proprioception & 1.75 & 1.34 & 2.18 \\
\hline
\end{tabular}

For example, Young Children are expected to make $88 \%$ more errors than Adults and $58 \%$ more errors than Older Children. Considering the Reality Environment, when using only Proprioception subjects are expected to make $92 \%$ more errors than when they rely only on Vision and $118 \%$ more errors than when using Vision + Proprioception. Considering the IVR Environment, when using only Proprioception subjects are expected to make $34 \%$ more errors than when they rely only on Vision and 35\% more errors than when using Vision + Proprioception. Moreover, comparing IVR to Reality Environment, subjects are expected to make $56 \%$ more errors when using only Vision and $75 \%$ more when using Vision + Proprioception.

\subsection{Model Fit}

Commonly, the model fit (i.e. the model's ability to explain the data) is assessed using statistical indexes as $R^{2}(\mathrm{Xu}, 2003)$. However, the usual definition of $R^{2}$ (i.e. the variance of the predicted values divided by the variance of the data) has some problems in the Bayesian framework because the numerator can be larger than the denominator (Gelman, Goodrich, Gabry, \& Vehtari, 2018). To overcome this issue, Gelman et al. (2018) proposed a Bayesian alternative definition of $R^{2}$ :

$$
\text { Bayesian } R^{2}=\frac{\text { Explained Variance }}{\text { Explained Variance }+ \text { Residual Variance }}=\frac{V_{n=1}^{N} \hat{y}_{n}}{V_{n=1}^{N} \hat{y}_{n}+V_{n=1}^{N} r_{n}}
$$

where $r_{n}=y_{n}-\hat{y_{n}}$ are the residuals of the fitted model. Given this definition, Bayesian $R^{2}$ is always between 0 and 1 by construction, and thus it follows the same interpretation as the common $R^{2}$ : an estimate of the proportion of variance explained.

The estimated value of Bayesian $R^{2}$ for the model m.2 is $0.26(95 \% B C I=0.19 ; 0.34)$, that is the model explains $26 \%$ of the variability of the data (Table 18 ). 
Table 18: Bayesian $R^{2}$

\begin{tabular}{ccccc}
\hline \multicolumn{2}{c}{ Bayesian $R^{2}$} & & \multicolumn{2}{c}{$\mathbf{9 5} \%$ BCI } \\
\cline { 1 - 1 } Estimate & $\mathbf{S E}$ & & Lower & Upper \\
\hline 0.26 & 0.038 & & 0.187 & 0.337 \\
\hline Note: & $n_{\text {subjects }}=49 ;$ & $n_{\text {observations }}=578$
\end{tabular}

Posterior predictive check is an alternative way to evaluate model goodness-of-fit (Kruschke, 2013). Qualitative visual inspection of the posterior predictive check allows us to assess whether data predicted by the model resemble the observed data or if the residuals between actual and simulated data follow certain patterns.

In 16 the data predicted by 100 sampled parameter vectors are presented. Visual inspection reveals a good fit of the model with the predicted data that properly and consistently follow the observed data.

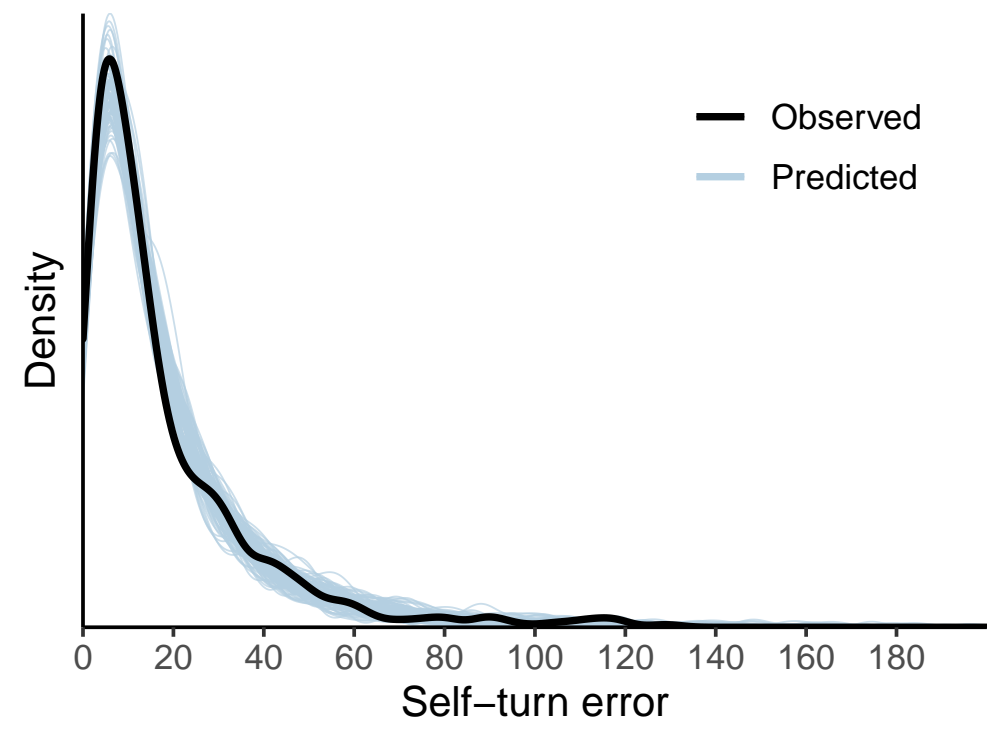

Figure 16: Posterior predictive check. The black line represents the distribution of observed data $\left(n_{\text {subjects }}=49 ; n_{\text {observations }}=578\right)$. The gray lines represent the distribution of predicted data for each of the sampled parameter vector $\left(n_{\text {samples }}=100\right)$. 


\section{Maximum Likelihood}

In this section all models are estimated with the traditional maximum likelihood approach using the $\mathrm{R}$ package lme4 (Bates et al., 2014). In several cases, convergence is not reached (i.e. unreliable results).

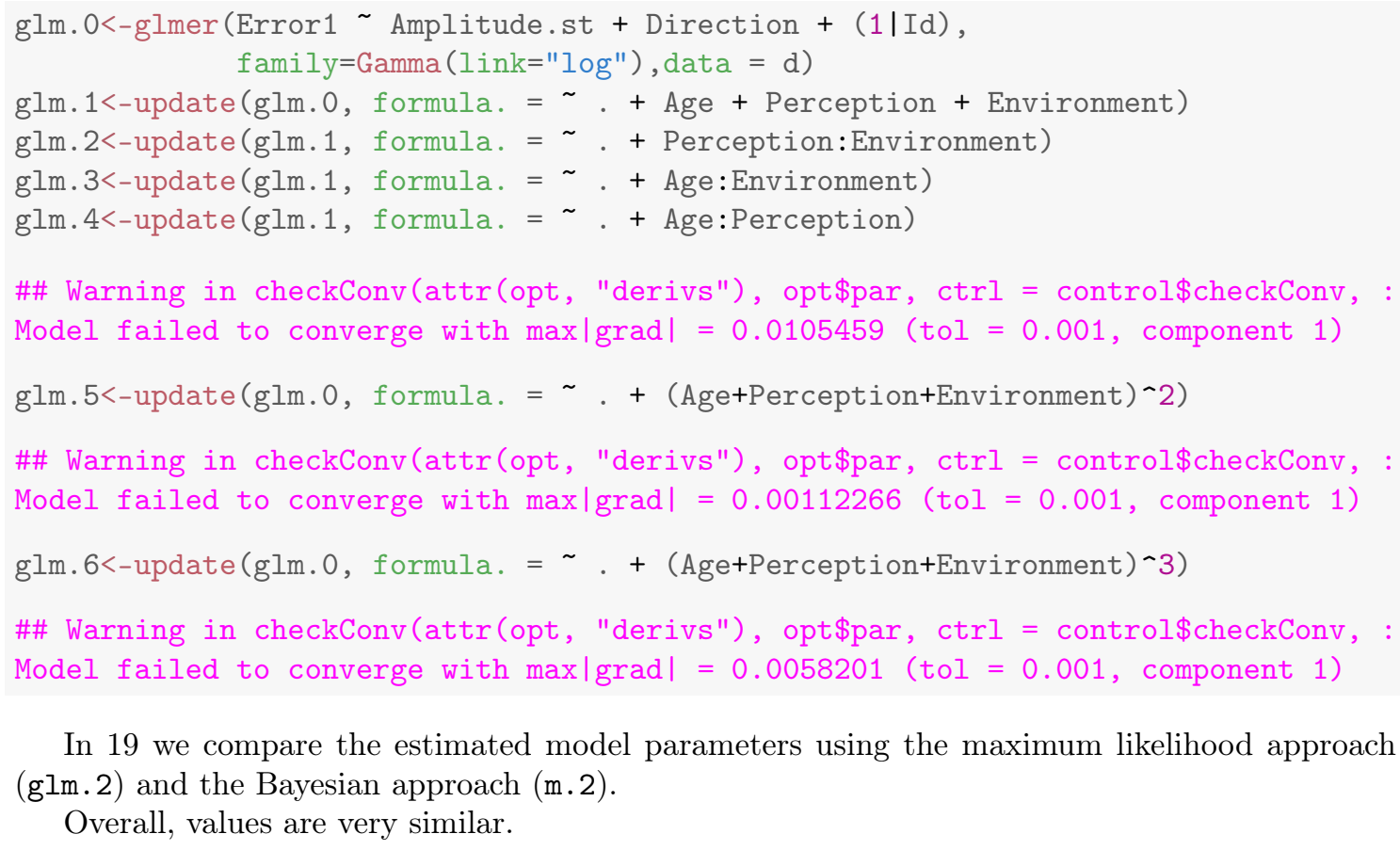

In 19 we compare the estimated model parameters using the maximum likelihood approach (glm.2) and the Bayesian approach (m.2).

Overall, values are very similar.

Table 19: Comparison between Bayesian m.2 and Maximum-Likelihood glm.2 models

\begin{tabular}{|c|c|c|c|c|}
\hline Parameters & \multicolumn{2}{|c|}{$\mathrm{m} .2$} & \multicolumn{2}{|c|}{ glm.2 } \\
\hline Name & Estimate & Est.Error & Estimate & Std.Error \\
\hline Intercept & 2.79 & 0.12 & 2.77 & 0.12 \\
\hline Amplitude.st & 0.22 & 0.04 & 0.22 & 0.04 \\
\hline Direction (left) & 0.10 & 0.07 & 0.09 & 0.07 \\
\hline Environment (IVR) & 0.08 & 0.12 & 0.07 & 0.12 \\
\hline Age & & & & \\
\hline Older Children & 0.18 & 0.13 & 0.17 & 0.14 \\
\hline Young Children & 0.62 & 0.13 & 0.62 & 0.14 \\
\hline Perception & & & & \\
\hline Vision & -0.64 & 0.12 & -0.65 & 0.12 \\
\hline Vision + Proprioception & -0.77 & 0.12 & -0.77 & 0.12 \\
\hline Interaction & & & & \\
\hline Vision : IVR & 0.36 & 0.17 & 0.37 & 0.17 \\
\hline $\begin{array}{r}\text { Vision }+ \text { Proprioception : } \\
\text { IVR }\end{array}$ & 0.48 & 0.17 & 0.48 & 0.17 \\
\hline
\end{tabular}

Note: Baseline category for Direction is "right". Baseline category for Age is "Adults". Baseline category for Perception is "Proprioception". Baseline category for Environment is "Reality". IVR = Immersive Virtual Reality. $n_{\text {subjects }}=$ $49 ; n_{\text {observations }}=578$ 


\section{Session Information}

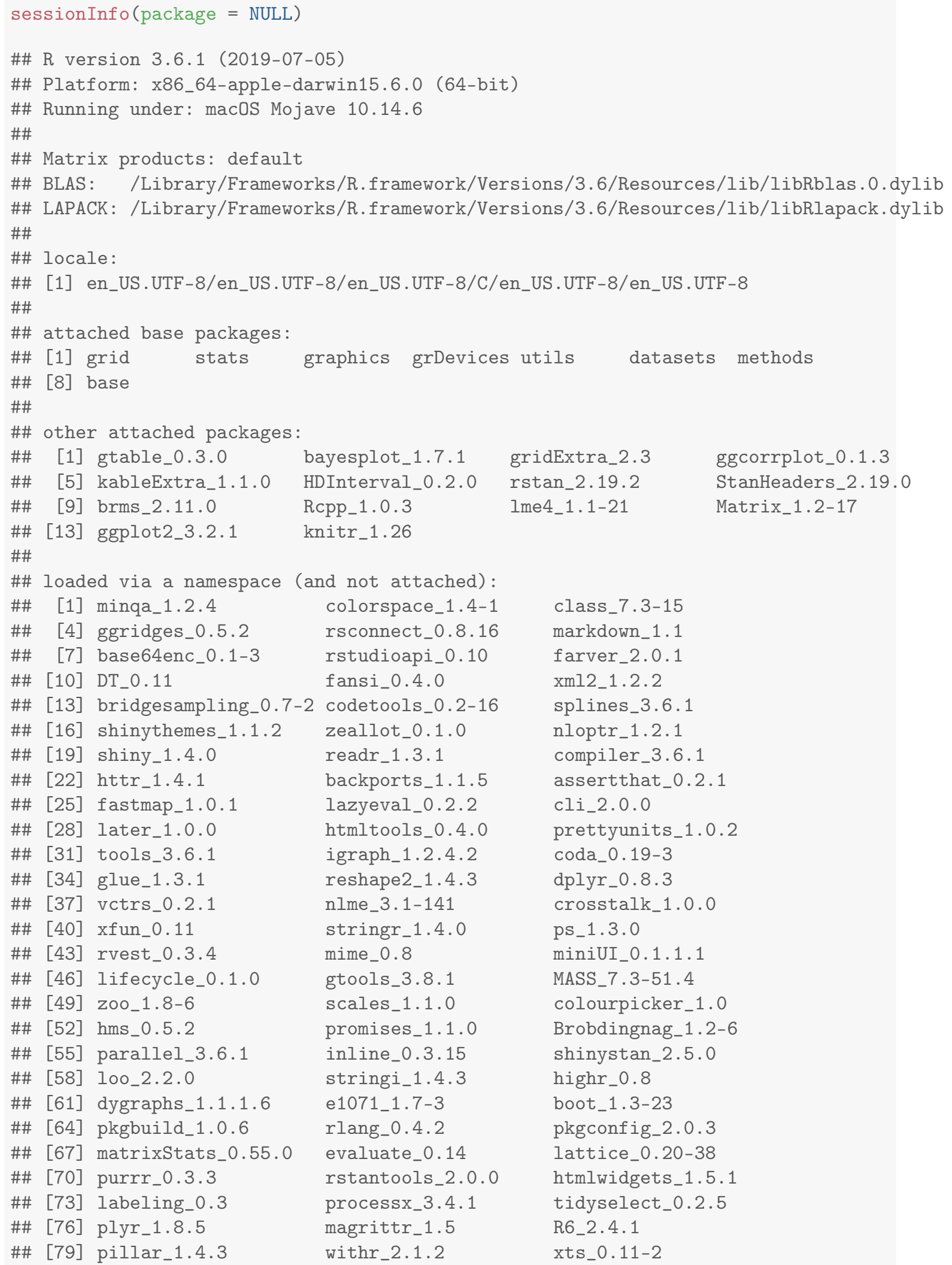


\#\# [82] abind_1.4-5

\#\# [85] rmarkdown_1.16

\#\# [88] digest_0.6.23

\#\# [91] httpuv_1.5.2

\#\# [94] viridisLite_0.3.0 tibble_2.1.3

callr_3.4.0

webshot_0.5.1

stats4_3.6.1

shinyjs_1.1 crayon_1.3.4

threejs_0.3.1

xtable_1.8-4

munsell_0.5.0 


\section{References}

Akaike, H. (1973). Information theory and an extension of the maximum likelihood principle. In Second International Symposium on Information Theory (pp. 267-281). Petrov, B. N. and Csaki, F., editors.

Bates, D., Mächler, M., Bolker, B., \& Walker, S. (2014). Fitting Linear Mixed-Effects Models using lme4. arXiv:1406.5823 [stat]. arXiv: 1406.5823 [stat]

Bolker, B., Brooks, M., Clark, C., Geange, S., Poulsen, J., Stevens, M., \& White, J. (2009). Generalized linear mixed models: A practical guide for ecology and evolution. Trends in Ecology ES Evolution, 24(3), 127-135. doi:10.1016/j.tree.2008.10.008

Bürkner, P.-C. (2017). Brms: An R package for Bayesian multilevel models using Stan. Journal of Statistical Software, $80(1), 1-28$. doi: $10.18637 /$ jss.v080.i01

Etz, A., \& Vandekerckhove, J. (2018). Introduction to Bayesian Inference for Psychology. Psychonomic Bulletin \& Review, 25(1), 5-34. doi 10.3758/s13423-017-1262-3

Fong, Y., Rue, H., \& Wakefield, J. (2010). Bayesian inference for generalized linear mixed models. Biostatistics, 11 (3), 397-412. doi 10.1093/biostatistics/kxp053

Fox, J. (2016). Applied regression analysis and generalized linear models (Third Edition). OCLC: ocn894301740. Los Angeles: SAGE.

Gelman, A., Carlin, J. B., Stern, H. S., Dunson, D. B., Vehtari, A., \& Rubin, D. B. (2013). Bayesian data analysis. doi:10.1201/b16018

Gelman, A., Goodrich, B., Gabry, J., \& Vehtari, A. (2018). R-squared for Bayesian regression models. The American Statistician, 1-6. doi $10.1080 / 00031305.2018 .1549100$

Gelman, A., Hwang, J., \& Vehtari, A. (2014). Understanding predictive information criteria for Bayesian models. Statistics and Computing, 24 (6), 997-1016. doi 10.1007/s11222-013-9416-2

Gelman, A., \& Rubin, D. B. (1992). Inference from iterative simulation using multiple sequences. Statistical Science, 7(4), 457-472.

Hoffman, M. D., \& Gelman, A. (2014). The No-U-Turn Sampler: Adaptively Setting Path Lengths in Hamiltonian Monte Carlo. Journal of Machine Learning Research, 15, 1593-1623. arXiv: 1111.4246

Kruschke, J. K. (2013). Posterior predictive checks can and should be Bayesian: Comment on Gelman and Shalizi, 'Philosophy and the practice of Bayesian statistics'. British Journal of Mathematical and Statistical Psychology, 66 (1), 45-56. doi 10.1111/j.2044-8317.2012.02063.x

Kruschke, J. K., \& Liddell, T. M. (2018). The Bayesian new statistics: Hypothesis testing, estimation, meta-analysis, and power analysis from a Bayesian perspective. Psychonomic Bulletin E3 Review, 25 (1), 178-206. doi 10.3758/s13423-016-1221-4

Lo, S., \& Andrews, S. (2015). To transform or not to transform: Using generalized linear mixed models to analyse reaction time data. Frontiers in Psychology, 6. doi $10.3389 /$ fpsyg.2015. 01171

McElreath, R. (2016). Statistical Rethinking: A Bayesian Course with Examples in $R$ and Stan (1st ed.). doi:10.1201/9781315372495

Neal, R. (2012). MCMC using Hamiltonian dynamics. In S. Brooks, A. Gelman, G. Jones, \& X. Meng (Eds.), Handbook of Markov chain Monte Carlo (pp. 113-162). http://arxiv.org/abs/1206.1901. New York, NY: Chapman.

Ng, V. K., \& Cribbie, R. A. (2017). Using the Gamma Generalized Linear Model for Modeling Continuous, Skewed and Heteroscedastic Outcomes in Psychology. Current Psychology, 36 (2), 225-235. doi:10.1007/s12144-015-9404-0

R Core Team. (2018). R: A language and environment for statistical computing. R Foundation for Statistical Computing. https://www.R-project.org/. Vienna, Austria.

Romeijn, J., \& van de Schoot, R. [R.]. (2008). A philosopher's view on Bayesian evaluation of informative hypotheses. In H. Hoijtink, I. Klugkist, \& P. Boelen (Eds.), Bayesian evaluation of informative hypotheses (pp. 329-357). doi:10.1007/978-0-387-09612-4_16

Stan Development Team. (2017a). Stan Modeling Language: User's Guide and Reference Manual. http://mc-stan.org/manual.html. 
Stan Development Team. (2017b). Stan: A C ++ library for probability and sampling. http://mcstan. org.

van de Schoot, R. [Rens], Kaplan, D., Denissen, J., Asendorpf, J. B., Neyer, F. J., \& van Aken, M. A. (2014). A gentle introduction to Bayesian analysis: Applications to developmental research. Child Development, 85(3), 842-860. doi $10.1111 /$ cdev.12169

Vandekerckhove, J., Matzke, D., \& Wagenmakers, E.-J. (2015). Model Comparison and the Principle of Parsimony (J. R. Busemeyer, Z. Wang, J. T. Townsend, \& A. Eidels, Eds.). Oxford University Press.

Vehtari, A., Gelman, A., \& Gabry, J. (2017). Practical Bayesian model evaluation using leave-oneout cross-validation and WAIC. Statistics and Computing, 27(5), 1413-1432. doi:10.1007/ s11222-016-9696-4

Wagenmakers, E.-J., \& Farrell, S. (2004). AIC model selection using Akaike weights. Psychonomic Bulletin \&s Review, 11(1), 192-196. doi 10.3758/BF03206482

$\mathrm{Xu}, \mathrm{R}$. (2003). Measuring explained variation in linear mixed effects models. Statistics in Medicine, (22), 3527-3541. doi: $10.1002 / \operatorname{sim} .1572$ 